\title{
Decentralized Sparsity-Based Multi-Source Association and State Tracking
}

\author{
Guohua Ren, Vasileios Maroulas and Ioannis D. Schizas ${ }^{1}$ \\ University of Texas at Arlington, Department of Electrical Engineering \\ 416 Yates Street, Arlington, TX 76015, USA \\ University of Tennessee at Knoxville, Department of Math \\ 1403 Circle Drive, Knoxville, TN 37996, USA
}

\begin{abstract}
The problem of tracking multiple sources using observations acquired at spatially scattered sensors is considered here. Two different sensing architectures are studied: i) A fusion-center based topology where sensors have a limited power budget; and ii) an ad hoc architecture where sensors collaborate with neighboring nodes enabling in-network processing. A novel source-to-sensor association scheme and tracking is introduced by enhancing the standard Kalman filtering minimization formulation with norm-one regularization terms. In the fusion-based topology a pertinent transmission power constraint is introduced, while coordinate descent techniques are employed to recover the unknown sparse observation matrix, select pertinent sensors and subsequently track the source states. In the ad hoc topology, the centralized minimization problem is written in a separable way and the alternating direction method of multipliers is utilized to construct an in-network algorithmic tracking and association framework. Numerical tests demonstrate that the resulting schemes are capable to associate sources with sensors, and track the unknown sources while adhering to any imposed power constraints.

Keywords: Sparsity; Multi-source tracking; Distributed processing; Sensor-to-source association
\end{abstract}

\footnotetext{
${ }^{1}$ Emails: \{guohua.ren@mavs.uta.edu,maroulas@math.utk.edu,schizas@uta.edu\} Work in this paper is supported by the NSF grant CCF 1218079, the AFOSR grant \#FA9550-15-1-0103, Simons Foundation Award \# 279870 and UTA.
}

Preprint submitted to Elsevier Signal Processing

September 3, 2015

(C) 2015. This manuscript version is made available under the Elsevier user license http://www.elsevier.com/open-access/userlicense/1.0/ 


\section{Introduction}

The task of tracking simultaneously many sources using sensor measurements at spatially scattered locations is extremely useful in a number of applications varying from surveillance to environmental monitoring [2]. The majority of existing tracking 5 approaches, such as the network schemes in $\lfloor 1,11,10,25,28,45,29]$, extend standard techniques such as Kalman filtering or particle filtering [3, 13]. The aforementioned approaches are developed under the assumption that the sensing model parameters are available. Such an assumption enables sensors to identify which sources they sense, which can further simplify the tracking process. However, in many settings it is not known which sensors observe each of the underlying field sources, while the signal attenuation from a source to a sensor is unavailable giving rise to an observation model with unknown parameters. In such settings source-to-sensor association is essential.

Alternative Kalman filtering schemes have been designed for settings where there is uncertainly in the state and observation model parameters which are known with some 15 additive error involved [24, 34]. A robust Kalman filter is developed in [42], which relies on the assumption that an uncertainty norm in the state and measurement models can be upper bounded. The latter work is extended in [43] to incorporate uncertainty with an upper bounded norm in the state and measurement noise covariance matrices. Robust Kalman filtering approaches have also been developed in sensor network settings. The work in [22] considers uncertainties in the measurement model introduced by an unknown sensor-to-fusion center channel. The channel follows a probabilistic on-off model, which is assumed known, and incorporated in the Kalman filter, to decide whether to use or drop measurements. Similarly, the work in [30] considers the design of Kalman filtering techniques in the presence of noise covariance matrix uncertainties with a bounded norm for a fusion-center based multisensor setting.

Different from these approaches, here no a priori information is available about the observation model parameter values, i.e., the sensing matrix entries. Availability of a model is crucial in associating the sources with the sensor measurements. Existing data association schemes [27, 14, 39, 16, 26] match observations with sources across time and rely on probabilistic models. Differently, the sensors-sources association scheme 
proposed here is relying only on the acquired sensor data and no probabilistic models are adopted. A different approach is followed in [37] where multiple fusion centers are present in the sensor network and evaluate the posterior Cramer-Rao lower bound that requires knowledge of the underlying data model. This type of bounds cannot be calculated in our setting. Data association of targets and measurements in the context of radar target tracking resort on probabilistic models to associate radar measurements acquired at different time instances with different targets or clutter [40, 41]. In our setting the association of sources and sensors will be performed both in space, due to the presence of scattered sensors, and time without the availability of a sensing model.

In practice, sources present in the monitored field are localized and affect only a small percentage of the sensors present in the sensor network (SN). For instance ground vibrating sources produce signals that undergo an exponential attenuation as they propagate in the ground. Such signals can be sensed in the measurements of sensors located a few meters away from the sources [17]. Interestingly, such a localized structured can be translated to a sensing matrix which has a large number of negligible (or zero) entries, i.e., a sparse matrix. Sparsity is exploited here to recover the unknown sparse sensing matrix in the measurement model, while tracking the different source states. To this end, norm-one regularization techniques, see e.g., [36, 46], will be employed to enhance the standard Kalman filter framework. The idea of sparsity has been exploited in the context of tracking [8, 38, 20], though the difference with respect to the present setting is that sparsity is in the source states and not in the sensing matrix.

Sparsity in the sensing matrix will be exploited here to jointly recover the sensing matrix and obtain tracking estimates for the, not necessarily sparse, field source states. The minimization formulation for the Kalman filter/smoother, see e.g., [3], will be enhanced with a pertinent norm-one regularization term. The sparsity-inducing terms will enable associating sources with sensors, and thus identify the sensors that acquire informative observations about the sources and use only those subsequently for tracking. Many existing tracking techniques require all sensors to be active [1, 45, 28, 29] which may be resource-consuming given the locality of the sources and the fact that only a few sensors bear information.

Two different network topologies of complementary nature are considered here. A 
fusion center (FC) based topology is considered first in which a fusion center is responsible for processing the sensor data and carrying out the association and tracking. Sparsity is combined with the introduction of a power constraint that enables utilization of a small percentage of sensors that are source-informative while a transmission power budget is not exceeded. The resulting novel constrained minimization formulation is tackled here via coordinate descent tools, see e.g., [4]. Power considerations have been incorporated in estimation and tracking [9], [19], though without taking into consideration issues such as source-to-sensor association and unknown model parameters.

The requirement for a more scalable and failure-relisient sensing architecture, while compromising computational speed, leads to tackle the novel norm-one regularized Kalman minimization framework in an ad hoc sensing topology. After reformulating the latter minimization problem in a separable form, the alternating direction method of multipliers (ADMM) combined with block coordinate descent, see e.g., [4, 5], is utilized to obtain an in-network algorithmic scheme that is capable of associating sensors with sources while tracking the source states.

The paper is organized as follows. The two different sensing topologies, along with the problem setting are outlined in Sec. 2 Building on the standard minimization formulation for the Kalman filter/smoother in [3], a pertinent norm-one regularization mechanism is used to jointly recover the sensing matrix, associate sources with sensors and track the source states. Further, power constraints are introduced to comply with a desired power transmission budget (Sec. 3). A separable formulation of the sparsityaware Kalman formulation and applicability of the ADMM toolbox is done in Sec. 4 resulting an effective in-network algorithm. Different from the preliminary work in [31] here i) a more refined formulation is provided; ii) ad hoc topologies are considered and distributed algorithms are derived; and iii) rigorous theoretical analysis accompanies the algorithmic construction process. A discussion about the communication and computational complexity can be found in Sec. [5] whereas extensive numerical tests studying the performance of the proposed framework are given in Sec. 7 


\section{Problem Statement}

Consider a field sensed by a total of $p$ sensors. Each sensor, say $j$, acquires scalar measurements $x_{j}(t)$ at time instant $t=0,1,2, \ldots$. Sensor observations contain information about $r$ underlying sources $s_{\rho}(t)$ which are represented by the scalar random variables $s_{\rho}(t)$, for $\rho=1, \ldots, r$. Source signals stacked in the state vector $95 \quad \mathbf{s}_{t}:=\left[s_{1}(t) \ldots s_{r}(t)\right]^{T}$ evolve according to the model:

$$
\mathbf{s}_{t}=\mathbf{F} \mathbf{s}_{t-1}+\mathbf{u}_{t}
$$

where $\mathbf{F}$ is the transition matrix, while $\mathbf{u}_{t}$ is the state noise assumed to be zero-mean Gaussian with covariance $\mathbf{Q}=\operatorname{diag}\left(\sigma_{u, 1}^{2}, \ldots, \sigma_{u, r}^{2}\right)$. To ensure the stability of the state vector, the spectral radius of $\mathbf{F}$ is assumed to be less than unity. Further, it is assumed here that the sources are uncorrelated with each other, i.e., $\mathbf{F}$ is a diagonal matrix whose

entries are less than one. Matrix $\mathbf{F}$ and the noise variances $\sigma_{u, \rho}^{2}$ are assumed available here, and can be found from e.g., the physics of the problem.

Each field source is observed by an unknown group of sensors. For instance, in Fig. 1 there are $r=2$ sources in the field. At time instant $t_{0}$, sensors $S_{1}, S_{2}, S_{4}$ (green region) acquire measurements about source $s_{1}\left(t_{0}\right)$, while source $s_{2}\left(t_{0}\right)$ is observed by sensors $S_{8}, S_{10}$ (red region). The rest of the sensors are just observing noise (blue sensors). Each sensor $S_{j}$ at time instance $t$ acquires a scalar measurement following the model

$$
x_{j}(t)=\sum_{\rho=1}^{r} h_{j, \rho}(t) s_{\rho}(t)+w_{j}(t) \text {, }
$$

where the coefficients $h_{j, \rho}(t)$ denote the unknown channel attenuation coefficient from source $s_{\rho}(t)$ to sensor $S_{j}$ at time $t$. Further, $w_{j}(t)$ is the corresponding zero-mean Gaussian sensing noise with variance $\sigma_{w}^{2}$. Such a setting could rise when tracking ground vibrating sources, see e.g., [17].

Specifically, let the $\rho$ th source represent a vibrating source while $s_{\rho}(t)$ corresponds to the vibration signal that the source generates on the ground with $\rho=1, \ldots, r$. These $r$ vibrating sources could correspond for instance to a moving vehicle, a person, animal or machinery, e.g., see [17]. The vibrating signals $s_{\rho}(t)$ in fact can be modeled in several scenaria, as autoregressive (AR) processes [see model in (1)] as advocated 
in [44]. Note that AR processes of higher order can be considered after employing vector states for each of the sources. For simplicity in exposition and clarity here AR-1 processes are considered.

Let $\mathbf{p}_{j}$ denote the fixed position of sensor $S_{j}$, while $\mathbf{l}_{\rho}(t)$ the location of the $\rho$ th source for $\rho=1, \ldots, r$. According to [17] the intensity of the vibrations measured at distance $d$ from the vibrating source are attenuated by a factor $e^{-\alpha d}$, where $\alpha$ a positive constant depending on the soil, e.g., for loess soil is $0.2 m^{-1}$ [17]. Applying the superposition principle, and assuming there is one dominant path of signal propagation from the source to the sensor it turns out that sensor $S_{j}$ acquires the measurement

$$
x_{j}(t)=\sum_{\rho=1}^{r} e^{-\alpha\left\|\mathbf{p}_{j}-\mathbf{l}_{\rho}(t)\right\|_{2}} s_{\rho}(t)+w_{j}(t) .
$$

125

It is clear from (3) that the channel attenuation coefficients are $h_{j, \rho}(t)=e^{-\alpha\left\|\mathbf{p}_{j}-\mathbf{l}_{\rho}(t)\right\|_{2}}$ and depend on the distance of the sensor from the source. Notice that among the summands $e^{-\alpha\left\|\mathbf{p}_{j}-\mathbf{l}_{\rho}(t)\right\|_{2}} s_{\rho}(t)$ some have very small amplitude when sensor $S_{j}$ is far from source $\rho$, while the amplitude increases as sensor $S_{j}$ is located closer to source $\rho$.

Thus, some of the attenuation coefficients may be close to zero depending on the distance between sources and sensors, e.g., in Fig. 1(left), sensor $S_{2}$ observes source $s_{1}(t)$, thus $h_{2,1}\left(t_{0}\right)$ is expected to be nonzero whereas $h_{2,2}\left(t_{0}\right)$ is negligible while sensor $S_{10}$ observes source $s_{2}(t)$, thus $h_{10,2}\left(t_{0}\right)$ is expected to be nonzero whereas $h_{10,1}\left(t_{0}\right)$ is negligible. Stacking the sensor measurements in $\mathbf{x}_{t}:=\left[x_{1}(t) \ldots x_{p}(t)\right]^{T}$ and the corresponding sensing noises in vector $\mathbf{w}_{t}:=\left[w_{1}(t), \ldots w_{p}(t)\right]^{T}$, the observation model can be summarized as

$$
\mathbf{x}_{t}=\mathbf{H}_{t} \mathbf{s}_{t}+\mathbf{w}_{t}
$$

where the observation matrix $\mathbf{H}_{t}$ contains the unknown attenuation coefficients $\mathbf{H}_{t}(j, \rho)$ $:=h_{j, \rho}(t)$. Note that the $\rho$ th column of the observation matrix $\mathbf{H}_{t}$ contains the attenuation coefficients between all sensors and source $s_{\rho}(t)$ at time $t$. For sensors close to the $\rho$ th vibrating source, the corresponding distances, $\left\|\mathbf{p}_{j}-\mathbf{l}_{\rho}(t)\right\|_{2}$, will be relatively small, thus leading to relatively large entries $\mathbf{H}_{t}(j, \rho)=e^{-\alpha\left\|\mathbf{p}_{j}-\mathbf{l}_{\rho}(t)\right\|_{2}}$, compared to sensors that are farther away. Since sources at a given instant $t$ are very localized and 
affect the measurements of a small percentage of sensors located a few meters away, many entries of $\mathbf{H}_{t}$ will be close to zero giving rise to an approximately sparse $\mathbf{H}_{t}$.

The field sources may be moving in space which results the time dependency of $\mathbf{H}_{t}$. As the sources move in the field, different group of sensors will observe them which results time-varying attenuation coefficients, resulting a time-varying $\mathbf{H}_{t}$. For example, in Fig. 1 at time instant $t_{0}$ source $s_{1}(t)$ is sensed by $S_{1}, S_{2}$ and $S_{4}$, while at time instant $t_{1}$ it is sensed by $S_{3}, S_{4}$. This further implies that the corresponding attenuation coefficients in the corresponding column of $\mathbf{H}_{t}$ will also change, e.g. in the first column of $\mathbf{H}_{t_{0}}$, entries $\mathbf{H}_{t_{0}}(1,1), \mathbf{H}_{t_{0}}(2,1)$ and $\mathbf{H}_{t_{0}}(4,1)$ have strong amplitude at $t_{0}$, whereas at $t_{1}$ they've switched to $\mathbf{H}_{t_{1}}(3,1)$ and $\mathbf{H}_{t_{1}}(4,1)$. Note that the support (indices of nonzero entries) of each column in $\mathbf{H}_{t}$, indicate which sensors acquire measurements about the source multiplying that column in (4).

Our goal here is to track the field source signals $\left\{s_{\rho}(t)\right\}_{\rho=1}^{r}$ using only sensor observations and the state model, but without having available the sensing matrix $\mathbf{H}_{t}$. For instance in the presence of vibrating sources, tracking the different signals can facilitate applications such as target classification, see e.g. [35, 23]. In the following, novel algorithms will be developed to jointly track the sparse matrix $\mathbf{H}_{t}$, while performing source-sensor association to track each of the sources using only those observations acquired at sensors observing a source. The proposed scheme will enable multi-source tracking by using only the source-informative sensors. The latter benefits can be seen in Fig. 1 in which it is obvious that the blue sensors do not carry any information and therefore there is no need for the FC to communicate with them. The aforementioned problem will be considered in two different sensor network topologies.

First, a fusion-center based topology, as the one depicted in Fig. 1, is considered in which every sensor only acquires measurements and if necessary transmits its observation to a fusion center responsible for carrying out multi-source tracking and sources-to-sensors association. The proposed tracking framework will reduce sensorto-FC communications, respect any power constraints across the network while using measurements from source-informative sensors to carry out the tracking.

The second topology considered, will be an ad hoc sensor network comprising of $p$ sensors. In the ad-hoc topology, sensors not only will acquire measurements but also 
will be responsible for carrying out information processing in an in-network fashion. Specifically, sensors will only communicate with their single-hop neighboring sensors who are within communication range. For instance, in Fig. 2, sensor $S_{6}$ is able to communicate with sensors $S_{3}, S_{4}, S_{7}$ and $S_{8}$ which form the single-hop neighborhood for $S_{6}$. The neighboring sensors of sensor $j$ are contained in the set $\mathcal{N}_{j}$, while the sensor network is modeled as an undirected graph and the inter-sensor links are assumed to be symmetric.

The same state and measurement models introduced in (1) and (4) will be considered here. The goal will be to identify the time-varying groups of sensors observing different sources by recovering the support of the unknown $\mathbf{H}_{t}$, while tracking $\mathbf{s}_{t}$. Since there is no fusion-center, in the ad-hoc setting, sensors will have to collaborate and exchange information with their single-hop neighbors to locally track the sensors and identify the different groups of informative sensors.

Remark: Note that the two different topologies considered here are complimentary, thus it is of interest to consider the problem of multi-source tracking in both the FCbased topology which enables fast processing/tracking since sensors send their data directly to a FC. The ad hoc topology involves iterative algorithms that may take longer time to converge, though this architecture offers robustness to failures and lack of a single point of failure, such as a FC.

\section{FC-Based Tracking and Sensor Selection}

We start with developing a proper joint tracking and source-to-sensor association algorithm for a FC-based setting. A pertinent minimization framework based on a novel blending of Kalman filtering/smoothing and sparsity-aware matrix recovery via normone regularization is utilized to jointly recover the sensing matrix $\mathbf{H}$, and track the states $\left\{\mathbf{s}_{\rho}(t)\right\}_{\rho=1}^{r}$ at the FC.

\subsection{Kalman Filtering Optimization}

Kalman filtering (KF) is an iterative filtering technique which updates the prediction and correction state estimates, namely $\hat{\mathbf{s}}(t \mid t-1):=E\left[\mathbf{s}(t) \mid \mathbf{x}_{0}, \ldots, \mathbf{x}_{t-1}\right]$, 
$\hat{\mathbf{s}}(t \mid t):=E\left[\mathbf{s}(t) \mid \mathbf{x}_{0}, \ldots, \mathbf{x}_{t}\right]$, as well as their corresponding error covariance matrices

$$
\begin{aligned}
& \mathbf{M}_{t \mid t-1}:=E\left[\left(\mathbf{s}_{t}-\hat{\mathbf{s}}(t \mid t-1)\right)\left(\mathbf{s}_{t}-\hat{\mathbf{s}}(t \mid t-1)\right)^{T}\right] \\
& \mathbf{M}_{t \mid t}:=E\left[\left(\mathbf{s}_{t}-\hat{\mathbf{s}}(t \mid t)\right)\left(\mathbf{s}_{t}-\hat{\mathbf{s}}(t \mid t)\right)^{T}\right]
\end{aligned}
$$

by utilizing all data acquired up to time $t$. Detailed KF recursions can be found in [21](Ch. 12). If the model parameters in (1) and (4) are available, the Kalman estimates for $K+1$ (where $K \geq 1$ ) consecutive states, say $\left\{\mathbf{s}_{t-K}, \ldots, \mathbf{s}_{t}\right\}$, can be formulated as the minimizer, see e.g., [3], of

$$
\left\{\check{\mathbf{s}}_{\tau}\right\}_{\tau=t-K}^{t}=\arg \min _{\mathbf{s}_{t-K}, \ldots, \mathbf{s}_{t}} J_{t, K}\left(\left\{\mathbf{s}_{\tau}, \mathbf{H}_{\tau}\right\}_{\tau=t-K}^{t}\right)
$$

where

$$
\begin{aligned}
& J_{t, K}\left(\left\{\mathbf{s}_{\tau}, \mathbf{H}_{\tau}\right\}_{\tau=t-K}^{t}\right):=\sum_{\tau=t-K+1}^{t}\left(\mathbf{x}_{\tau}-\mathbf{H}_{\tau} \mathbf{s}_{\tau}\right)^{T} \mathbf{\Sigma}_{w}^{-1}\left(\mathbf{x}_{\tau}-\mathbf{H}_{\tau} \mathbf{s}_{\tau}\right) \\
& +\sum_{\tau=t-K+1}^{t}\left(\mathbf{s}_{\tau}-\mathbf{F} \mathbf{s}_{\tau-1}\right)^{T} \mathbf{Q}^{-1}\left(\mathbf{s}_{\tau}-\mathbf{F} \mathbf{s}_{\tau-1}\right) \\
& +\left(\mathbf{s}_{t-K}-\hat{\mathbf{s}}_{t-K}\right)^{T} \mathbf{M}_{t-K \mid t-K}^{-1}\left(\mathbf{s}_{t-K}-\hat{\mathbf{s}}_{t-K}\right),
\end{aligned}
$$

where $\boldsymbol{\Sigma}_{w}:=\sigma_{w}^{2} \cdot \mathbf{I}$ contains the sensing noise variances and $\hat{\mathbf{s}}_{t-K}$ corresponds to an estimate of the state $\mathbf{s}_{t-K}$ and $\mathbf{M}_{t-K \mid t-K}$ the corresponding covariance at time instant $t-K$ using sensor data $\mathbf{x}_{0}, \ldots, \mathbf{x}_{t-K}$.

Traditionally, see e.g., [21], the cost in (7) is minimized w.r.t the states assuming that $\mathbf{H}_{\tau}$ is given. If $K=1$, the standard $\mathrm{KF}$ recursions are obtained by minimizing (7), namely $\check{\mathbf{s}}_{\tau}=\hat{\mathbf{s}}[\tau \mid \tau]$. Notice that when $K>1$, the estimates $\check{\mathbf{s}}_{t}$ correspond to the state estimates obtained by a fixed-lag ( $K$ lags here) Kalman smoother, see e.g., [3, pg. 177], i.e., $\check{\mathbf{s}}_{\tau}=\hat{\mathbf{s}}(\tau \mid t)$ for $\tau=t-K, \ldots, t$.

Assuming that the sensing matrix $\mathbf{H}_{t}$ is available, the minimization problem is performed with respect to the unknown sources' states $\left\{\mathbf{s}_{\tau}\right\}_{\tau=t-K}^{t}$. However, the sensing matrix itself is unknown in the considered setting and need to be found as well. The formulation in (7) does not account for the sparse unknown structure of the sensing matrix $\mathbf{H}_{t}$. To this end, and inspired by existing work in $\ell_{1}$-regularization, see e.g., [36], we introduce a norm-one regularization term in (7) and obtain the minimization 
formulation

$$
\begin{aligned}
\min _{\left\{\mathbf{s}_{\tau}\right\}_{\tau=t-K}^{t}, \mathbf{H}_{\tau}} & \sum_{\tau=t-K+1}^{t}\left(\mathbf{x}_{\tau}-\mathbf{H}_{\tau} \mathbf{s}_{\tau}\right)^{T} \mathbf{\Sigma}_{w}^{-1}\left(\mathbf{x}_{\tau}-\mathbf{H}_{\tau} \mathbf{s}_{\tau}\right)+\sum_{\rho=1, \tau=t-K+1}^{r, t} \lambda_{\rho}\left\|\mathbf{h}_{\tau, \rho}\right\|_{1} \\
& +\sum_{\tau=t-K+1}^{t}\left(\mathbf{s}_{\tau}-\mathbf{F} \mathbf{s}_{\tau-1}\right)^{T} \mathbf{Q}^{-1}\left(\mathbf{s}_{\tau}-\mathbf{F} \mathbf{s}_{\tau-1}\right) \\
& +\left(\mathbf{s}_{t-K}-\hat{\mathbf{s}}_{t-K}\right)^{T} \mathbf{M}_{t-K \mid t-K}^{-1}\left(\mathbf{s}_{t-K}-\hat{\mathbf{s}}_{t-K}\right)
\end{aligned}
$$

where $\|\cdot\|_{1}$ denotes the one-norm, while $\mathbf{h}_{\tau, \rho}$ corresponds to the $\rho$ th column of matrix $\mathbf{H}_{\tau}$. The weights $\lambda_{\rho}$ are nonnegative sparsity-controlling coefficients that control the number of nonzeros in the column of $\mathbf{h}_{\tau, \rho}$. Tackling the optimization problem in (8)

will allow i) recover the missing observation matrix $\mathbf{H}_{\tau}$ and perform sources-to-sensors association; ii) track the unknown $r$ sources present in the monitored field.

\subsection{Multi-source State Tracking}

Aiming at minimizing the cost in (8), we resort to block coordinate descent technique where we minimize (8) wrt the states $\left\{\mathbf{s}_{\tau}\right\}_{\tau=t-K}^{t}$ by fixing the matrices $\mathbf{H}_{\tau}$ to their most recent update. Further, to simplify $[8]$, the time index of $\mathbf{H}_{t}$ is removed by assuming that $\mathbf{H}$ is varying very slowly during $K$ consecutive time instances. Then, the cost is minimized wrt $\mathbf{H}_{t}$ while fixing the states to their most up-to-date values. This section focuses on updating the states while fixing $\mathbf{H}$.

Denote $\hat{\mathbf{H}}_{t-1}$ as the estimate for $\mathbf{H}$ obtained during the time interval $[t-K-1, t-$ 1]. By fixing $\mathbf{H}$ to $\hat{\mathbf{H}}_{t-1}$, the cost in $(8)$ is optimized w.r.t the states $\left\{\mathbf{s}_{\tau}\right\}_{\tau=t-K}^{t}$. After differentiating the cost in (8) w.r.t the augmented state vector $\mathbf{s}_{\alpha, t}:=\left[\mathbf{s}_{t-K}^{T} \ldots \mathbf{s}_{t}^{T}\right]^{T}$ and setting the derivative equal zero, we will get the following state estimates (See Appendix A):

$$
\check{\mathbf{s}}_{\alpha, t}:=\left[\check{\mathbf{s}}_{t-K, t}^{T}, \ldots, \check{\mathbf{s}}_{t, t}^{T}\right]^{T}=\left(\mathbf{F}_{\alpha}^{T} \mathbf{Q}_{\alpha}^{-1} \mathbf{F}_{\alpha}\right)^{-1} \mathbf{F}_{\alpha}^{T} \mathbf{Q}_{\alpha}^{-1} \mathbf{x}_{\alpha, t}
$$

where

$$
\begin{aligned}
& \mathbf{Q}_{\alpha, t}:=\operatorname{bdiag}(\mathbf{M}_{t-K \mid t-K}, \overbrace{\mathbf{Q}, \ldots, \mathbf{Q}}^{K \text { times }}, \overbrace{\mathbf{\Sigma}_{w}, \ldots, \mathbf{\Sigma}_{w}}^{K \text { times }}), \\
& \mathbf{x}_{\alpha, t}:=\left[-\hat{\mathbf{s}}_{t-K}^{T}, \mathbf{0}^{T}, \ldots, \mathbf{0}^{T}, \mathbf{x}_{t-K+1}^{T}, \ldots, \mathbf{x}_{t}^{T}\right]^{T}
\end{aligned}
$$


The state estimate $\hat{\mathbf{s}}_{t-K}$ is provided by a local Kalman filter run at the FC with the estimated $\hat{\mathbf{H}}_{t}$ and the available sensor measurements in $\mathbf{x}_{t-K}$. Matrices $\mathbf{Q}_{\alpha, t}$ and $\mathbf{x}_{\alpha, t}$ have sizes $[K p+(K+1) r] \times[K p+(K+1) r]$ and $[(K+1) r+K p] \times 1$ respectively, while matrix $\mathbf{F}_{\alpha}:=\left[\begin{array}{ll}\mathbf{F}_{1}^{T} & \mathbf{F}_{2, t}^{T}\end{array}\right]^{T}$ has size $(K+1) r+K p \times(K+1) r$ and formed as:

$$
\mathbf{F}_{1}=\left[\begin{array}{cccc}
-\mathbf{I}_{r \times r} & \mathbf{0} & \ldots & \mathbf{0} \\
\mathbf{F} & -\mathbf{I}_{r \times r} & \ldots & \mathbf{0} \\
\vdots & \vdots & \ddots & \vdots \\
\mathbf{0} & \ldots & \mathbf{F} & -\mathbf{I}_{r \times r}
\end{array}\right], \mathbf{F}_{2, t}=\left[\begin{array}{ccccc}
\mathbf{0} & \hat{\mathbf{H}}_{t-1} & \mathbf{0} & \ldots & \mathbf{0} \\
\mathbf{0} & \mathbf{0} & \hat{\mathbf{H}}_{t-1} & \ldots & \mathbf{0} \\
\vdots & \vdots & \ddots & & \vdots \\
\mathbf{0} & \mathbf{0} & \mathbf{0} & \ldots & \hat{\mathbf{H}}_{t-1}
\end{array}\right] .
$$

It is worth mentioning that matrix $\left(\mathbf{F}_{\alpha}^{T} \mathbf{Q}_{\alpha}^{-1} \mathbf{F}_{\alpha}\right)^{-1}$ is block tri-diagonal. In order to

solve the system of linear equations in (97, there exist efficient approaches, see e.g. [15, pg. 174] whose computational complexity is linearly increasing with $K$.

\subsection{Sparse Sensing Matrix Recovery}

As stated earlier, the task of performing real-time sources-to-sensors association turns out to boil down to the problem of recovering the support of the sensing matrix $\mathbf{H}_{t}$. To this end, the cost $(8)$ will be minimized wrt to $\mathbf{H}$ by fixing the states to their latest updated estimates $\left\{\check{\mathbf{s}}_{\tau, t}\right\}_{\tau=t-K}^{t}$ obtained in (9). A coordinate descent way is utilized to minimize (8) wrt one entry of $\mathbf{H}$ while fixing the other terms to their most recent values. At time $t$, let $\hat{\mathbf{H}}_{t}^{\varkappa}(i, \rho)$ denote the coordinate update for entry $\mathbf{H}(i, \rho)$ obtained during coordinate cycle $\varkappa$ for all $i=1, \ldots, p$ and $\rho=1, \ldots, r$. When updating $\mathbf{H}(i, j)$, all remaining entries of $\mathbf{H}$ are fixed to the previous coordinate cycle updates, namely $\hat{\mathbf{H}}_{t}^{\varkappa-1}(i, \ell)$, and as shown in $\operatorname{Apdx}$. B $\hat{\mathbf{H}}_{t}^{\varkappa}(i, \rho)$ is obtained as:

$$
\left.\hat{\mathbf{H}}_{t}^{\varkappa}(i, \rho):=\arg \min _{h} \sigma_{w}^{-2} \| \mathbf{y}_{i, t}^{\varkappa-1}-\check{\mathbf{s}}_{\rho, t} \cdot h\right) \|_{2}^{2}+\lambda_{\rho}|h|
$$

where $\mathbf{y}_{i, t}^{\varkappa-1}$ is a $K \times 1$ vector with entries

$$
\mathbf{y}_{i, t}^{\varkappa-1}(m)=x_{i}(t-K+m)-\sum_{\ell=1, \ell \neq \rho}^{r} \hat{\mathbf{H}}_{t}^{\varkappa-1}(i, \ell) \check{\mathbf{s}}_{t-K+m, t}(\ell)
$$

for $m=1, \ldots, K$ and $\check{\mathbf{s}}_{\rho, t}:=\left[\check{\mathbf{s}}_{t-K+1, t}(\rho), \ldots, \check{\mathbf{s}}_{t, t}(\rho)\right]^{T}$. The minimization in (13) boils down to a sparse regression (Lasso) problem involving a scalar. It turns out (see 
Apdx. B) that the minimizer in (13) is:

$$
\hat{\mathbf{H}}_{t}^{\varkappa}(i, \rho)=\operatorname{sgn}\left(\check{\mathbf{s}}_{\rho, t}^{T} \mathbf{y}_{i, t}^{\varkappa-1}\right) \cdot\left(\frac{\check{\mathbf{s}}_{\rho, t}^{T} \mathbf{y}_{i, t}^{\varkappa-1}}{\left\|\check{\mathbf{s}}_{\rho, t}\right\|_{2}^{2}}-\frac{\lambda_{\rho}}{2 \sigma_{w}^{-2}\left\|\check{\mathbf{s}}_{\rho, t}\right\|_{2}^{2}}\right)_{+}
$$

where $(z)_{+}=\max (z, 0)$. Note that warm starts are employed at every time instant $t$, where $\hat{\mathbf{H}}_{t}^{0}(i, \ell)=\hat{\mathbf{H}}_{t-1}^{C}(i, \ell)$ for $i=1, \ldots, p$ and $\ell=1, \ldots, r . C$ is the number of coordinate cycles applied at every time instant $t$. A stopping criterion applied for the coordinate method is the following: For two consecutive updating cycles, a cost difference, using the cost in (8), is evaluated. If the cost difference becomes lower than a predefined threshold, say $\epsilon$, then the updating process is terminated automatically.

\subsection{Transmission Power Budget Constraints}

It is often the case that sensors have to operate under a stringent power budget, imposed here in the form of a constraint in the total transmission power that all sensors can consume. Thus, it is of interest to introduce in the formulation in (8) a mechanism respecting such a power constraint.

Starting from the formulation at $(8)$, we introduce in the cost in (8) selection variables to decide which sensors' data participate, or not, while ensuring the total transmission power does not exceed a desired level $P_{t o t}$. In detail:

$$
\begin{aligned}
& \arg \underset{\mathbf{s}_{t-K}, \ldots, \mathbf{s}_{t}}{\min } J_{t, K}\left(\mathbf{D},\left\{\mathbf{s}_{\tau}\right\}_{\tau=t-K}^{t}, \mathbf{H}\right) \\
& \text { s. to } \sum_{j=1}^{p} d_{j} \sigma_{x, j}^{2}<P_{t o t}, \text { and } d_{j} \in[0,1], \quad j=1, \ldots, p
\end{aligned}
$$

where

$$
\begin{aligned}
& J_{t, K}\left(\mathbf{D},\left\{\mathbf{s}_{\tau}\right\}_{\tau=t-K}^{t}, \mathbf{H}\right):=\sum_{\tau=t-K+1}^{t}\left(\mathbf{x}_{\tau}-\mathbf{H} \mathbf{s}_{\tau}\right)^{T} \mathbf{D} \mathbf{\Sigma}_{w}^{-1}\left(\mathbf{x}_{\tau}-\mathbf{H} \mathbf{s}_{\tau}\right) \\
& +\sum_{\tau=t-K+1}^{t}\left(\mathbf{s}_{\tau}-\mathbf{F} \mathbf{s}_{\tau-1}\right)^{T} \mathbf{Q}^{-1}\left(\mathbf{s}_{\tau}-\mathbf{F} \mathbf{s}_{\tau-1}\right) \\
& +\left(\mathbf{s}_{t-K}-\hat{\mathbf{s}}_{t-K}\right)^{T} \mathbf{M}_{t-K \mid t-K}^{-1}\left(\mathbf{s}_{t-K}-\hat{\mathbf{s}}_{t-K}\right)+\sum_{\rho=1}^{r} \lambda_{\rho}\left\|\mathbf{h}_{\rho}\right\|_{1},
\end{aligned}
$$

where $\boldsymbol{\Sigma}_{w}:=\sigma_{w}^{2} \cdot \mathbf{I}_{p \times p}$, while $\hat{\mathbf{s}}_{t-K}$ corresponds to an estimate of the state $\mathbf{s}_{t-K}$ and $\mathbf{M}_{t-K \mid t-K}$ denotes the corresponding covariance acquired as described in Sec. 
3.2. $\mathbf{D}$ is the diagonal matrix whose diagonal entries $d_{j}$ lie in the interval $[0,1]$, and control which sensor measurements will participate in tracking the source states in $\mathbf{s}_{t}$ during the interval $[t-K+1, t]$. $\mathbf{D}$ will be selected such that the prediction error is minimized while ensuring that the transmission power will not exceed $P_{t o t}$. Note that signal variance in a way quantifies the power of the signal and therefore the amount of power needed to transmit it, thus the summation $\sum_{j=1}^{P} d_{j} \sigma_{x, j}^{2}$ quantifies the average power needed to transmit data from the selected sensors to the FC. However, it should be emphasized that this constraint does not account for communication parameters such as the channel.

We resort to block coordinate descent techniques, see e.g. [4], where we minimize (17) w.r.t. the entries of $\mathbf{D}$, or $\mathbf{H}$ or $\left\{\mathbf{s}_{\tau}\right\}_{\tau=t-K+1}^{t}$ while fixing the others to their latest update. Fixing the state vectors and $\mathbf{H}$ to their latest updates, the minimization of (17) w.r.t. the diagonal coefficients $d_{j}$ in $\mathbf{D}$ corresponds to

$$
\begin{aligned}
& \left\{\hat{d}_{1, t}, \hat{d}_{2, t}, \ldots, \hat{d}_{p, t}\right\}:=\arg \min \sum_{\tau=t-K+1}^{t} \sum_{j=1}^{p} d_{j} \sigma_{w}^{-2}\left[x_{j}(\tau)-\left[\hat{\mathbf{H}}_{t-1}\right]_{j} \check{\mathbf{s}}_{\tau, t-1}\right]^{2} \\
& \text { s. to } \sum_{j=1}^{p} d_{j} \sigma_{x, j}^{2}<P_{t o t}, \text { and } d_{j} \in[0,1], \quad j=1, \ldots, p
\end{aligned}
$$

where the sensor observation variances can be estimated as

$$
\left(\hat{\sigma}_{x, 1, t-1}^{2}, \ldots, \hat{\sigma}_{x, \rho, t-1}^{2}\right)=\operatorname{diag}\left(\hat{\mathbf{H}}_{t-1} \hat{\boldsymbol{\Sigma}}_{s} \hat{\mathbf{H}}_{t-1}^{T}\right)
$$

while the source covariance estimate $\hat{\boldsymbol{\Sigma}}_{s}$ can be estimated as $\hat{\boldsymbol{\Sigma}}_{s}=\hat{\mathbf{s}}_{\tau, t-1} \cdot \hat{\mathbf{s}}_{\tau, t-1}^{T}$. Further, $\left[\hat{\mathbf{H}}_{t-1}\right]_{j}$ : denotes the $j$ th row of $\hat{\mathbf{H}}_{t-1}$, while $\check{\mathbf{s}}_{\tau, t-1}$ corresponds to the state estimates obtained from minimizing the cost $J_{t-1, K}$, and $\hat{\mathbf{H}}_{t-1}$ is the sensing matrix estimate obtained during time interval $[t-K, t-1]$. The minimization problem in (18) corresponds to a linear program with linear constraints and can be solved, e.g., using the interior point method, see e.g. [6]. After the coefficients $\hat{d}_{j, t}$ are determined in (18) for the time interval $[t-K+1, t]$, they are compared with a threshold. If $\hat{d}_{j, t}$ is larger than the threshold, then it is set to $\breve{d}_{j, t}=1$ and the FC pings the corresponding sensor to transmit its measurements, otherwise $\hat{d}_{j, t}$ is set to $\check{d}_{j, t}=0$ and the corresponding sensor remains silent. The threshold is selected as the smallest value in the interval $(0.5,1)$ such that the total power consumed by the selected sensors, namely 
$\sum_{j=1}^{p}\left(\check{d}_{j, t} \sigma_{x_{j}}^{2}\right)$, does not exceed the power budget $P_{t o t}$. The left end of the interval 0.5 is set such that any sensor $j$ with $\hat{d}_{j, t}>0.5$ is considered a candidate sensor for being selected. Further, the smallest value in the interval $(0.5,1)$ allows the largest number of sensors to be selected while not exceeding the power budget $P_{t o t}$.

Once $\hat{d}_{j, t}$ are determined and set to 1 or 0 as described earlier, estimates of the sources' state vectors $\left\{\mathbf{s}_{\tau}\right\}_{\tau=t-K+1}^{t}$, and recovery of the sparse $\mathbf{H}_{t}$ can be done in the same way as described in Section 3 . Note that since some $\breve{d}_{j, t}$ are zero, corresponding summands in the first term of (17) will not be participating in the updating of the state vectors and $\mathbf{H}$ via (9) and (15), respectively.

\subsection{Algorithmic Summary}

To initialize the matrix $\mathbf{H}_{t}$, at time instant $t=0$, and obtain an estimate of the number of sources in the monitored field, the sparse decomposition scheme in [33], summarized in Sec. 3.6 can be applied. To collect a sufficiently large number of data while ensuring that source states do not change significantly, as required to apply [33], a sufficiently fast sampling rate can be applied at a start-up phase to ensure the aforementioned requirement. The processing takes place at the FC which is responsible for tracking $\left\{d_{j, t}\right\}_{j=1}^{p}, \mathbf{H}_{t}$ and $\mathbf{s}_{t}$.

Once the sensor selection variables $\hat{d}_{j, t}$ are found via solving (18), they are compared with a threshold. Then, depending on whether $\hat{d}_{j, t}$ is larger or smaller than the threshold, is set to $\check{d}_{j, t}=1$ or $\check{d}_{j, t}=0$, respectively. Then, the state vectors $\left\{\mathbf{s}_{\tau}\right\}_{\tau=t-K+1}^{t}$ and $\mathbf{H}_{t}$ are estimated, by solving the costs in (17) after keeping only these summands within the first term of (17) with index $j$ for which $\breve{d}_{j, t}=1$.

At the start-up stage all sensors transmit their data to the FC such that the state vectors, $\mathbf{H}_{0}$ and $d_{j, 0}$ 's are initialized. Then, throughout the execution stage of the algorithm, only the sensors with $\breve{d}_{j, t}=1$ will transmit their data into the FC. This

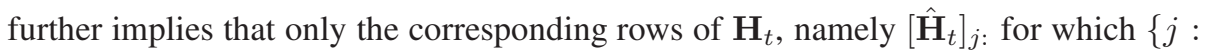
$\left.\check{d}_{j, t}=1\right\}$ will be updated.

Since the sources may be moving, there is a need to update $\hat{d}_{j, t}$ on a continuous basis. The approach followed here is to re-update the weights using the start-up phase steps every, say $\Omega$, time instances. For slowly varying sources, the time interval sep- 
arating two updates of the $d_{j}$ weights can be increased and the reconfiguration step does not have to be applied very frequently. Thus, a small percentage of sensors will be sending data to the FC most of the time. In the simulation we quantify the percentage of active sensors and the associated tracking error. In the FC-based architecture, sensors are just sensing measurements and get pinged (if $\breve{d}_{j, t}=1$ ) by the FC to transmit their data. Note that if $P_{t o t}>\sum_{j=1}^{p} \sigma_{x, j}^{2}$, then all $d_{j}$ 's can be 1 and the sensors selection step is not really needed. The approach is summarized in the Algorithm 1 Table.

3.6. Sparsity-aware Matrix Decomposition via [33]

The scheme in [33] focuses on estimating the sparse observation matrix $\mathbf{H}_{0}$ in (4) at $t=0$, by decomposing into sparse factors the data covariance matrix

$$
\boldsymbol{\Sigma}_{x}=\mathbf{H}_{0} \boldsymbol{\Sigma}_{s} \mathbf{H}_{0}^{T}+\sigma_{w}^{2} \mathbf{I}_{p}=\overline{\mathbf{H}}_{0} \overline{\mathbf{H}}_{0}^{T}+\sigma_{w}^{2} \mathbf{I}_{p},
$$

where $\Sigma_{s}$ corresponds to the diagonal covariance of the entries in the state vector $\mathbf{s}_{0}$, while $\overline{\mathbf{H}}_{0}:=\mathbf{H}_{0} \boldsymbol{\Sigma}_{s}^{1 / 2}$. Further, let

$$
\mathbf{M}_{x}:=\boldsymbol{\Sigma}_{x}-\sigma_{w}^{2} \mathbf{I}_{p}=\overline{\mathbf{H}}_{0} \overline{\mathbf{H}}_{0}^{T}
$$

which corresponds to the signal covariance matrix after removing the impact of the noise covariance from the data covariance matrix. Note that the support of $\mathbf{H}$ and $\overline{\mathbf{H}}$ is the same since $\boldsymbol{\Sigma}_{s}$ is diagonal in structure. The covariance $\mathbf{M}_{x}$ at $t=0$ can be estimated by employing fast sampling during the initialization stage to ensure that the source state is essentially time-invariant, and then employing sample-averaging of the acquired measurements and removal of the noise variance. Let $\hat{\mathbf{M}}_{x}=\hat{\mathbf{\Sigma}}_{x}-\sigma_{w}^{2} \mathbf{I}$ be the estimator of $\mathbf{M}_{x}$, where $\hat{\boldsymbol{\Sigma}}_{x}$ corresponds to the sample-average covariance estimate.

An estimate of the sparse matrix $\mathbf{H}_{0}$ is found in [33] by resorting to the sparsityinducing minimization formulation

$$
\hat{\mathbf{H}}_{0}=\arg \min _{\mathbf{H}}\left\|\hat{\mathbf{M}}_{x}-\mathbf{H H}^{T}\right\|_{F}^{2}+\sum_{\rho=1}^{r} \lambda_{\rho}\left\|\mathbf{h}_{\rho}\right\|_{1}
$$

where $\|\cdot\|_{1}$ refers to norm-one and $\lambda_{\rho}$ is the sparsity-controlling coefficients for the $\rho$ th column of matrix $\mathbf{H}$, namely $\mathbf{h}_{\rho}$. The minimization problem in (22) is tackled via a 
coordinate decent approach where the cost in (22) is cyclically minimized with respect to an entry of $\mathbf{H}$ while keeping the rest entries fixed. Details on the recursive updating formulas involved in minimizing (22) can be found in [33].

\section{Distributed Tracking and Sensor Selection}

Our goal is to develop a joint multi-source association and tracking algorithm that is distributed, and can be implemented in an ad hoc network of spatially distributed sensors. Sensors placed in an ad hoc configuration can both acquire and process data.

\subsection{Separable Minimization}

Since there is not a central FC in the ad hoc setting, there is a need to rewrite the centralized cost in (7) in a way that takes into account the network topology. The reason for this step is to derive subsequently an algorithm that involves information exchanges only between single-hop neighbors that are sufficiently close to communicate. Toward this end, the cost in (7) will be splitted into local subcosts which can be tackled in parallel and locally across sensors, while proper equality constraints will be used to account for the network topology (communication graph).

Local vectors $\mathbf{s}_{\tau, j}$ for $j=1, \ldots, p$ are introduced to indicate local optimization variables stored and updated at sensor $j$. The vectors $\mathbf{s}_{\tau, j}$ corresponds to a local version of state vector $\mathbf{s}_{\tau}$, at sensor $j$. The local variables $\mathbf{s}_{\tau, j}$ are introduced to allow every sensor $j$ to keep tracking $\mathbf{s}_{\tau}$. Since all the vectors $\left\{\mathbf{s}_{\tau, j}\right\}_{j=1}^{p}$ correspond to local copies of $\mathbf{s}_{\tau}$, they should be equal. Thus, the equality constraint $\mathbf{s}_{\tau, 1}=\mathbf{s}_{\tau, 2}=\ldots=\mathbf{s}_{\tau, p}$ needs to accompany a minimization formulation that will rely on the local vectors $\mathbf{s}_{\tau, j}$.

However, such a constraint is challenging to satisfy since it requires data communication between any possible pair of sensors in the network (full connectivity). In order to comply with the network topology, each local state $\mathbf{s}_{\tau, j}$ is required to be equal to the local state vectors update in its neighborhood $\mathcal{N}_{j}$, i.e., $\mathbf{s}_{\tau, j}=\mathbf{s}_{\tau, j^{\prime}}$ for every sensor $j^{\prime} \in \mathcal{N}_{j}$. Connectivity of the network communication graph ensures that the previous single-neighborhood constraints guarantee that $\mathbf{s}_{\tau, 1}=\mathbf{s}_{\tau, 2}=\ldots=\mathbf{s}_{\tau, p}$ 
$\forall \tau=0,1,2, \ldots$ Then, the cost in 8 can be equivalently rewritten as:

$$
\begin{aligned}
J_{t, K}\left(\left\{\mathbf{s}_{\tau, j}\right\}_{\tau=t-K}^{t}, \mathbf{H}\right) & :=\sum_{j=1}^{p} \sum_{\tau=t-K+1}^{t}\left(x_{j}(\tau)-\mathbf{H}_{j:} \mathbf{s}_{\tau, j}\right)^{2} \sigma_{w}^{-2} \\
& +\frac{1}{p} \sum_{j=1}^{p} \sum_{\tau=t-K+1}^{t}\left(\mathbf{s}_{\tau, j}-\mathbf{F} \mathbf{s}_{\tau-1, j}\right)^{T} \mathbf{Q}^{-1}\left(\mathbf{s}_{\tau, j}-\mathbf{F} \mathbf{s}_{\tau-1, j}\right) \\
+ & \frac{1}{p} \sum_{j=1}^{p}\left(\mathbf{s}_{t-K, j}-\hat{\mathbf{s}}_{t-K, j}\right)^{T} \mathbf{M}_{t-K \mid t-K, j}^{-1}\left(\mathbf{s}_{t-K, j}-\hat{\mathbf{s}}_{t-K, j}\right)+\sum_{\rho=1}^{r} \lambda_{\rho}\left\|\mathbf{h}_{\rho}\right\|_{1},
\end{aligned}
$$

The cost in (23) can be further rewritten as (details in Appendix C):

$$
J_{t, K}\left(\left\{\mathbf{s}_{\tau, j}\right\}_{\tau=t-K}^{t}, \mathbf{H}\right):=\sum_{j=1}^{p} \sum_{\tau=t-K}^{t}\left\|\boldsymbol{\xi}_{\tau, j}-\mathbf{G}_{\tau, j} \mathbf{s}_{\tau, j}\right\|_{2}^{2}+\sum_{\rho=1}^{r} \lambda_{\rho}\left\|\mathbf{h}_{\rho}\right\|_{1}
$$

where

$\xi_{1}(\tau)=\left\{\begin{array}{cc}1 & \tau>t-K \\ 0 & \tau=t-K\end{array}, \quad\right.$ and $\xi_{2}(\tau)=\left\{\begin{array}{ll}0 & \tau>t-K \\ 1 & \tau=t-K\end{array}\right.$,

while

$\boldsymbol{\xi}_{\tau, j}=\left[\begin{array}{c}\xi_{1}(\tau) \sigma_{w}^{-1} x_{j}(\tau) \\ \xi_{1}(\tau)(p \mathbf{Q})^{-\frac{1}{2}} \mathbf{F} \mathbf{s}_{\tau-1, j} \\ \xi_{2}(\tau) p^{-1 / 2} \mathbf{M}_{t-K \mid t-K, j}^{-\frac{1}{2}} \hat{\mathbf{s}}_{t-K, j}\end{array}\right]$ and $\mathbf{G}_{\tau, j}=\left[\begin{array}{c}\xi_{1}(\tau) \sigma_{w}^{-1} \mathbf{H}_{j:} \\ \xi_{1}(\tau)(p \mathbf{Q})^{-\frac{1}{2}} \\ \xi_{2}(\tau) p^{-1 / 2} \mathbf{M}_{t-K \mid t-K, j}^{-\frac{1}{2}}\end{array}\right]$.

The state estimate $\hat{\mathbf{s}}_{t-K, j}$ can be updated at each sensor by running a local Kalman

filter utilizing its neighboring sensors' measurements along with its own measurement at $t-K$, while $\hat{\mathbf{M}}_{t-K \mid t-K, j}$ denotes the corresponding local covariance matrix. After incorporating the local equality constraints introduced earlier, the minimization formulation in (8) is reformulated in a separate way as follows:

$$
\begin{aligned}
& \arg \min _{\left\{\mathbf{s}_{\tau, j}\right\}_{\tau=t-K, j=1}^{t, p}, \mathbf{H}} \sum_{j=1}^{p} \sum_{\tau=t-K}^{t}\left\|\boldsymbol{\xi}_{\tau, j}-\mathbf{G}_{\tau, j} \mathbf{s}_{\tau, j}\right\|_{2}^{2}+\sum_{\rho=1}^{r} \lambda_{\rho}\left\|\mathbf{h}_{\rho}\right\|_{1}, \\
& \text { s. to } \mathbf{s}_{\tau, j}=\mathbf{s}_{\tau, j^{\prime}}, \text { for } j^{\prime} \in \mathcal{N}_{j}, j=1, \ldots, p \text { and } \tau=t-K, \ldots, t
\end{aligned}
$$

As in the FC-based setting, the time index of the sensing matrix $\mathbf{H}$ has been removed, since it is treated fixed during the time window $[t-K, t]$. Note that the minimization formulation in (8) and (25) are equivalent in the sense that they have the same minimizers for the state vectors and $\mathbf{H}$. 
In order to perform joint multi-source tracking and source-to-sensor association, it is required to solve (25) in a distributed fashion. An alternating scheme will be employed, where we optimize (25) w.r.t the entries of $\mathbf{H}$ while fixing the state, and vice versa.

\subsection{Algorithmic Construction}

To minimize (25) in a fully distributed fashion w.r.t the state vectors $\mathbf{s}_{\tau . j}$, we employ the alternating direction method of multipliers (ADMM), see e.g., [7] [5]. To facilitate applicability of ADMM, we introduce a set of auxiliary variables $\mathbf{z}_{\tau, j}^{j^{\prime}}$ for $j^{\prime} \in \mathcal{N}_{j}$ and $\tau=t-K, \ldots, t$. These auxiliary variables will be used to derive the local updating recursions across sensors and will finally be eliminated. These variables are used to substitute the constraints in 25] with the equivalent ones:

$$
\mathbf{s}_{\tau, j}=\mathbf{z}_{\tau, j}^{j^{\prime}} \text { and } \mathbf{s}_{\tau, j}=\mathbf{z}_{\tau, j^{\prime}}^{j}, \text { for } j^{\prime} \in \mathcal{N}_{j} \text { and } j \neq j^{\prime}
$$

Necessary in utilizing the ADMM to tackle (25) is the augmented Lagrangian function, see, e.g. [7, Chapt 3], which here takes the form

$$
\begin{aligned}
\mathcal{L}_{a}\left[\left\{\mathbf{s}_{\tau, j}\right\}, \mathbf{v}, \boldsymbol{\omega}\right] & =\sum_{j=1}^{p} \sum_{\tau=t-K}^{t}\left\|\boldsymbol{\xi}_{\tau, j}-\mathbf{G}_{\tau, j} \mathbf{s}_{\tau, j}\right\|_{2}^{2}+\sum_{\rho=1}^{r} \lambda_{\rho}\left\|\mathbf{h}_{\rho}\right\|_{1} \\
& +\sum_{j=1}^{p} \sum_{j^{\prime} \in \mathcal{N}_{j}} \sum_{\tau=t-K}^{t}\left[\left(\mathbf{v}_{\tau, j}^{j^{\prime}}\right)^{T}\left(\mathbf{s}_{\tau, j}-\mathbf{z}_{\tau, j}^{j^{\prime}}\right)+\left(\boldsymbol{\omega}_{\tau, j}^{j^{\prime}}\right)^{T}\left(\mathbf{s}_{\tau, j}-\mathbf{z}_{\tau, j^{\prime}}^{j}\right)\right] \\
& +0.5 c \sum_{j=1}^{p} \sum_{j^{\prime} \in \mathcal{N}_{j}} \sum_{\tau=t-K}^{t}\left[\left\|\mathbf{s}_{\tau, j}-\mathbf{z}_{\tau, j}^{j^{\prime}}\right\|_{2}^{2}+\left\|\mathbf{s}_{\tau, j}-\mathbf{z}_{\tau, j^{\prime}}^{j}\right\|_{2}^{2}\right]
\end{aligned}
$$

The Lagrangian multipliers $\mathbf{v}_{\tau, j}^{j^{\prime}}$ 's and $\boldsymbol{\omega}_{\tau, j}^{j^{\prime}}$ correspond to the constraints $\mathbf{s}_{\tau, j}=\mathbf{z}_{\tau, j}^{j^{\prime}}$ and $\mathbf{s}_{\tau, j}=\mathbf{z}_{\tau, j^{\prime}}^{j}$, respectively. Further, $c$ is a positive coefficient, while the vectors $\mathbf{v}$ and $\boldsymbol{\omega}$ contain the multipliers $\mathbf{v}_{\tau, j}^{j^{\prime}}$ and $\boldsymbol{\omega}_{\tau, j}^{j^{\prime}}$ that will make sure that the equality constraints $\mathbf{s}_{\tau, j}=\mathbf{z}_{\tau, j}^{j^{\prime}}$ and $\mathbf{s}_{\tau, j}=\mathbf{z}_{\tau, j^{\prime}}^{j}$ are satisfied when minimizing 25].

Due to the nonconvex structure of the cost in 27, we employ block coordinate descent. First, 27) is minimized w.r.t $\mathbf{H}$ while treating the states $\mathbf{s}_{\tau, j}$ as fixed. Then, after fixing $\mathbf{H}$ the cost in (27) will be minimized w.r.t $\mathbf{s}_{\tau, j}$ subject to the constraints (26).

\section{Minimization of 27) w.r.t $\mathbf{H}$ :}


Let $k=0,1,2, \ldots, T$ denote the coordinate descent cycle index when tackling (24). Every sensor $j$ is responsible for updating the $j$ th row of $\mathbf{H}$, namely the entries $\{\mathbf{H}(j, \rho)\}_{\rho=1}^{r}$. To this end, the local states $\mathbf{s}_{\tau, j}$ will be set to their most recent updates $\hat{\mathbf{s}}_{\tau, j}^{k+1}$ which corresponds to the ADMM outcome at the end of coordinate cycle $k+1$ (details later). From the augmented Lagrangian function in 24) it turns out (using similar arguments as in Apdx. B).

$$
\left.\hat{\mathbf{H}}_{t}^{k+1}(j, \rho):=\arg \min _{h} \sigma_{w}^{-2} \| \mathbf{y}_{j, t}^{k+1}-\hat{\mathbf{s}}_{t, j}^{\rho, k} \cdot h\right) \|_{2}^{2}+\lambda_{\rho}|h|,
$$

where $\hat{\mathbf{s}}_{t, j}^{\rho, k}:=\left[\hat{\mathbf{s}}_{t-K+1, j}^{k}(\rho), \hat{\mathbf{s}}_{t-K+2, j}^{k}(\rho), \ldots, \hat{\mathbf{s}}_{t, j}^{k}(\rho)\right]^{T}$ and $\mathbf{y}_{j, t}^{k+1}$ is a $K \times 1$ vector with entries

$$
\mathbf{y}_{j, t}^{k+1}(m)=x_{j}(t-K+m)-\sum_{\ell=1, \ell \neq \rho}^{r} \hat{\mathbf{H}}_{t}^{k}(j, \ell) \hat{\mathbf{s}}_{t-K+m, j}^{k}(\ell), m=1, \ldots, M,
$$

where $\hat{\mathbf{s}}_{t-K+m, j}^{k}(\ell)$ correspond to the $\ell$ th entry of the state estimates $\hat{\mathbf{s}}_{t-K+m, j}^{k}$ obtained via $\mathrm{ADMM}$ as described next at the end of coordinate cycle $k$. Using similar arguments as in Apdx. B, it turns out that the optimal solution in 280 is:

$$
\hat{\mathbf{H}}_{t}^{k+1}(j, \rho)=\operatorname{sgn}\left(\left[\hat{\mathbf{s}}_{t, j}^{\rho, k}\right]^{T} \mathbf{y}_{j, t}^{k+1}\right) \times\left(\frac{\left[\hat{\mathbf{s}}_{t, j}^{\rho, k}\right]^{T} \mathbf{y}_{j, t}^{k+1}}{\left\|\hat{\mathbf{s}}_{t, j}^{\rho, k}\right\|_{2}^{2}}-\frac{\lambda_{\rho}}{2 \sigma_{w, j}^{-2}\left\|\hat{\mathbf{s}}_{t, j}^{\rho, k}\right\|_{2}^{2}}\right)_{+} .
$$

355

Sensor $j$ can update $\hat{\mathbf{H}}_{t}^{k+1}(j, \rho)$, for $\rho=1, \ldots, r$, locally since both $\mathbf{y}_{j, t}^{k+1}$ and $\hat{\mathbf{s}}_{t, j}^{\rho, k}$ can be formed using its local measurements $\mathbf{x}_{j}(\tau)$ and the local estimates $\hat{\mathbf{s}}_{\tau, j}^{k}$.

ADMM-based minimization of (27) w.r.t $\mathbf{s}_{\tau, j}$ and $\mathbf{z}_{\tau, j}^{j^{\prime}}$ 's:

Here $C$ ADMM iterations are applied per coordinate cycle $k+1$ to obtain across sensors estimates for the states $\mathbf{s}_{\tau, j}$ for the time window $\tau=t-K, \ldots, t$. ADMM involves the following three steps (detailed later):

i) Update the Lagrange multipliers using gradient ascent iterations. Let $\mathbf{v}_{\tau, j}^{j^{\prime}, k+1}(\varkappa)$ and $\boldsymbol{\omega}_{\tau, j}^{j^{\prime}, k+1}(\varkappa)$ denote the ADMM updates for the multipliers $\mathbf{v}_{\tau, j}^{j^{\prime}}$ and $\boldsymbol{\omega}_{\tau, j}^{j^{\prime}}$, where the positive integer $\varkappa$ denotes the ADMM iteration index performed during coordinate cycle $k+1$. Here $C$ ADMM iterations are nested within coordinate cycle $k+1$.

ii) Minimize the augmented Lagrangian function in 27 w.r.t $\mathbf{s}_{\tau, j}$ for $j=1, \ldots, p$, while fixing $\mathbf{z}_{\tau, j}^{j^{\prime}}, \mathbf{v}_{\tau, j}^{j^{\prime}}$ and $\boldsymbol{\omega}_{\tau, j}^{j^{\prime}}$ to their most up-to-date values, namely $\mathbf{z}_{\tau, j}^{j^{\prime}, k+1}(\varkappa)$, $\mathbf{v}_{\tau, j}^{j^{\prime}, k+1}(\varkappa)$ and $\boldsymbol{\omega}_{\tau, j}^{j^{\prime}, k+1}(\varkappa)$ obtained at step i) to obtain update $\mathbf{s}_{\tau, j}^{k+1}(\varkappa+1)$. 
iii) Minimize the augmented Lagrangian function in 27 w.r.t the variables $\mathbf{z}_{\tau, j}^{j^{\prime}}$ while fixing $\mathbf{s}_{\tau, j}, \mathbf{v}_{\tau, j}^{j^{\prime}}$ and $\boldsymbol{\omega}_{\tau, j}^{j^{\prime}}$ to their most up-to-date values $\mathbf{s}_{\tau, j}^{k+1}(\varkappa)$ obtained at step ii), and $\mathbf{v}_{\tau, j}^{j^{\prime}, k+1}(\varkappa), \omega_{\tau, j}^{j^{\prime}, k+1}(\varkappa)$ obtained at step i).

Specifically, the first step of applying ADMM, during coordinate descent cycle $\varkappa+1$, is to update the multipliers using the gradient ascent iterations:

$$
\begin{aligned}
& \mathbf{v}_{\tau, j}^{j^{\prime}, k+1}(\varkappa)=\mathbf{v}_{\tau, j}^{j^{\prime}, k+1}(\varkappa-1)+c\left(\mathbf{s}_{\tau, j}^{k+1}(\varkappa)-\mathbf{z}_{\tau, j}^{j^{\prime}, k+1}(\varkappa)\right) \\
& \boldsymbol{\omega}_{\tau, j}^{j^{\prime}, k+1}(\varkappa)=\boldsymbol{\omega}_{\tau, j}^{j^{\prime}, k+1}(\varkappa-1)+c\left(\mathbf{s}_{\tau, j}^{k+1}(\varkappa)-\mathbf{z}_{\tau, j^{\prime}}^{j, k+1}(\varkappa)\right)
\end{aligned}
$$

for $j^{\prime} \in \mathcal{N}_{j}$, and $\tau=t-K, \ldots, t$. Warm starts are employed to initialize the Lagrange multipliers before applying $C$ ADMM iterations, namely $\mathbf{v}_{\tau, j}^{j^{\prime}, k+1}(0)=\mathbf{v}_{\tau, j}^{j^{\prime}, k}(C)$. The same procedure is followed to update and initialize the variables $\mathbf{s}_{\tau, j}, \mathbf{z}_{\tau, j}^{j^{\prime}}$ and $\boldsymbol{\omega}_{\tau, j}^{j^{\prime}}$.

The second step involves minimizing the augmented Lagrangian in (27) w.r.t. $\mathbf{s}_{\tau, j}$, while fixing the other variables to their most up-to-date values, namely $\left[\hat{\mathbf{H}}_{t}^{k+1}\right]_{j}$ : $(j$-th row of $\left.\hat{\mathbf{H}}_{t}^{k+1}\right), \mathbf{z}_{\tau, j}^{j^{\prime}, k+1}(\varkappa), \mathbf{v}_{\tau, j}^{j^{\prime}, k+1}(\varkappa)$ and $\omega_{\tau, j}^{j^{\prime}, k+1}(\varkappa)$. Then, as established in Apdx. $\mathrm{D}$, the update $\mathbf{s}_{\tau, j}^{k+1}(\varkappa+1)$ is formed as:

$$
\begin{aligned}
& \mathbf{s}_{\tau, j}^{k+1}(\varkappa+1)=\left(2 \mathbf{G}_{\tau, j}^{T} \mathbf{G}_{\tau, j}+2 c\left|\mathcal{N}_{j}\right| \mathbf{I}_{r \times r}\right)^{-1} \\
& \quad \times\left[2 \mathbf{G}_{\tau, j}^{T} \boldsymbol{\xi}_{\tau, j}-\sum_{j^{\prime} \in \mathcal{N}_{j}}\left(\mathbf{v}_{\tau, j}^{j^{\prime}, k+1}(\varkappa)+\boldsymbol{\omega}_{\tau, j}^{j^{\prime}, k+1}(\varkappa)\right)+c \sum_{j^{\prime} \in \mathcal{N}_{j}}\left(\mathbf{z}_{\tau, j}^{j^{\prime}, k+1}(\varkappa)+\mathbf{z}_{\tau, j^{\prime}}^{j, k+1}(\varkappa)\right)\right]
\end{aligned}
$$

The third ADMM step entails minimization of 27) w.r.t $\mathbf{z}_{\tau, j}^{j^{\prime}}$, while fixing the remaining variables to their most recent updates, namely $\hat{\mathbf{H}}_{t}^{k+1}, \mathbf{s}_{\tau, j}^{k+1}(\varkappa+1) \mathbf{v}_{\tau, j}^{j^{\prime}, k+1}(\varkappa)$ and $\boldsymbol{\omega}_{\tau, j}^{j^{\prime}, k+1}(\varkappa)$. Then, in Apdx. D it is shown that

$$
\begin{aligned}
\mathbf{z}_{\tau, j}^{j^{\prime}, k+1}(\varkappa+1) & =\frac{1}{2}\left[\mathbf{s}_{\tau, j}^{k+1}(\varkappa+1)+\mathbf{s}_{\tau, j^{\prime}}^{k+1}(\varkappa+1)\right] \\
& +\frac{1}{2 c}\left[\mathbf{v}_{\tau, j}^{j^{\prime}, k+1}(\varkappa)+\boldsymbol{\omega}_{\tau, j^{\prime}}^{j, k+1}(\varkappa)\right], \text { for } j=1, \ldots, p \text { and } j^{\prime} \in \mathcal{N}_{j} .
\end{aligned}
$$

In fact, the Lagrangian multipliers $\mathbf{v}_{\tau, j}^{j^{\prime}, k+1}(\varkappa)$ and $\omega_{\tau, j}^{j^{\prime}, k+1}(\varkappa)$ can be initialized randomly without affecting the convergence of the three ADMM steps as $\varkappa \rightarrow \infty$, see e.g., [32]. Substituting (34) into (31), and initializing the multipliers such that $\mathbf{v}_{\tau, j}^{j^{\prime}, k}(0)=-\boldsymbol{\omega}_{\tau, j^{\prime}}^{j, k}(0)$, then for all $\tau$ and $\varkappa=1, \ldots, C$, it holds $\mathbf{v}_{\tau, j}^{j^{\prime}, k}(\varkappa)=-\boldsymbol{\omega}_{\tau, j^{\prime}}^{j, k}(\varkappa)$ from which the first updating formulate in (31) can rewritten as

$$
\mathbf{v}_{\tau, j}^{j^{\prime}, k+1}(\varkappa)=\mathbf{v}_{\tau, j}^{j^{\prime}, k+1}(\varkappa-1)+0.5 c\left(\mathbf{s}_{\tau, j}^{k+1}(\varkappa)-\mathbf{s}_{\tau, j^{\prime}}^{k+1}(\varkappa)\right)
$$


Thus, there is no need to update and store $\boldsymbol{\omega}_{\tau, j^{\prime}}^{j, k}(\varkappa)$, since $\mathbf{v}_{\tau, j}^{j^{\prime}, k}(\varkappa)=-\boldsymbol{\omega}_{\tau, j^{\prime}}^{j, k}(\varkappa)$. Sensor $j$ updates the Lagrange multipliers $\left\{\mathbf{v}_{\tau, j}^{j^{\prime}, k+1}(\varkappa)\right\}_{j^{\prime} \in \mathcal{N}_{j}}$ for $\tau=t-K, \ldots, t$ and the local state vectors $\mathbf{s}_{\tau, j}$ whose updating formula in (33) can be written as

$$
\begin{aligned}
& \mathbf{s}_{\tau, j}^{k+1}(\varkappa+1)=\left(2 \mathbf{G}_{\tau, j}^{T} \mathbf{G}_{\tau, j}+2 c\left|\mathcal{N}_{j}\right| \mathbf{I}_{r \times r}\right)^{-1} \\
& \quad \times\left[2 \mathbf{G}_{j, \tau}^{T} \boldsymbol{\xi}_{\tau, j}-\sum_{j^{\prime} \in \mathcal{N}_{j}}\left(\mathbf{v}_{\tau, j}^{j^{\prime}, k+1}(\varkappa)-\mathbf{v}_{\tau, j^{\prime}}^{j, k+1}(\varkappa)\right)+c \sum_{j^{\prime} \in \mathcal{N}_{j}}\left(\mathbf{s}_{\tau, j}^{j^{\prime}, k+1}(\varkappa)+\mathbf{s}_{\tau, j^{\prime}}^{j, k+1}(\varkappa)\right)\right]
\end{aligned}
$$

where it can be clearly seen that the auxiliary variables $\mathbf{z}_{\tau, j}^{j^{\prime}}$ 's have been eliminated.

Recursions (35) and (36) constitute the novel distributed approach where each sensor $j$ just have to keep track of the multipliers $\left\{\mathbf{v}_{\tau, j}^{j^{\prime}}\right\}_{j^{\prime} \in \mathcal{N}_{j}}$ and the state updates $\mathbf{s}_{\tau, j}$ for $\tau=0, \ldots, t$. The initial values of $\mathbf{v}_{\tau, j}^{j^{\prime}}$ can be set to 0 , while the initial values of the s's will be obtained from sensor $j$ 's local Kalman filter prediction obtained using only its own measurements. In this way, all the sensors exchange information to their singlehop neighboring sensors. The convergence results in [32] ensure that if the number of ADMM iterations $C \rightarrow \infty$ during coordinate cycle $k+1$, then the local variables $\mathbf{s}_{\tau, j}$ become equal and $\lim _{\varkappa \rightarrow \infty} \mathbf{s}_{\tau, j}^{k+1}(\varkappa)$ converge to the state estimates in 9 , when $\mathbf{H}$ is set to $\hat{\mathbf{H}}_{t}^{k+1}$ in the corresponding quantities used to form them in (12).

\section{Computational and Communication Cost}

During time instant $t$, the augmented Lagrangian function in (27) is minimized by applying $T$ coordinate descent cycles. Once new measurements have been collected across sensors, namely $\mathbf{x}_{j}(t)$ for $j=1,2, \ldots, p$, the following process takes place. During coordinate cycle $k+1$, sensor $j$ first updates the $j$ th row of $\mathbf{H}$, and evaluates $\left\{\hat{\mathbf{H}}_{t}^{k+1}(j, \rho)\right\}_{\rho=1}^{r}$ via (30) using its local state updates $\hat{\mathbf{s}}_{t-K+m, j}^{k}$ for $m=1, \ldots, K$, the measurements $\mathbf{x}_{j}(t-k+1), \ldots, \mathbf{x}_{j}(t)$ and latest coordinate updates $\hat{\mathbf{H}}_{t}^{k}(j, \ell), \ell=$ 1 and $\ell \neq \rho$. Then, sensor $j$ runs $C$ ADMM iterations by carrying out (35) and (36). In detail, during ADMM iteration $\varkappa+1$, inside coordinate cycle $k+1$, sensor $j$ receives the $r \times 1$ state vectors $\mathbf{s}_{\tau, j}^{k+1}(\varkappa)$ from its neighbors $j^{\prime} \in \mathcal{N}_{j}$, and updates its Lagrange multipliers $\mathbf{v}_{\tau, j}^{j^{\prime}, k+1}(\varkappa)$ via (35).

Sensor $j$ receives the multiplier $\mathbf{v}_{\tau, j^{\prime}}^{j, k+1}(\varkappa)$ from neighbors $j^{\prime} \in \mathcal{N}_{j}$ which are used along with $\left\{\mathbf{s}_{\tau, j^{\prime}}^{k+1}(\varkappa)\right\}_{j^{\prime} \in \mathcal{N}_{j}}$ to update $\mathbf{s}_{\tau, j}^{k+1}(\varkappa+1)$ via $[36$. The steps are summarized 
in Alg. 2 Table.

During one ADMM iteration, sensor $j$ receives $\left\{\mathbf{v}_{\tau, j^{\prime}}^{j, k+1}(\varkappa), \mathbf{s}_{\tau, j^{\prime}}^{k+1}(\varkappa)\right\}_{\tau=t-K}^{t}$ from its neighbors $j^{\prime} \in \mathcal{N}_{j}$, which corresponds to $2 r(K+1)\left|\mathcal{N}_{j}\right|$ scalars. Since per time instant $t$, there are $T$ coordinate cycles with $C$ nested ADMM iterations each, the total scalars received during time instant $t$ are $2 r(K+1) C T\left|\mathcal{N}_{j}\right|$. Further, sensor $j$ has to transmit its local state vector estimate $\mathbf{s}_{\tau, j}^{k+1}(\varkappa)$ and multipliers $\left\{\mathbf{v}_{\tau, j}^{j^{\prime}, k+1}(\varkappa)\right\}_{j^{\prime} \in \mathcal{N}_{j}}$ which corresponds to $r\left(\left|\mathcal{N}_{j}\right|+1\right)(K+1)$ scalars. Thus, during time instant $t$ sensor $j$ transmits $r(K+1)\left(\left|\mathcal{N}_{j}\right|+1\right) C T$ scalars. Thus, the communication cost is proportional to the number of sources $r$, the neighborhood size and the number of coordinate cycles, as well as ADMM iterations applied per time instant $t$. Although the proposed algorithm is not scalable with respect to the number of sources, it has to be emphasized that the number of sources in practice is smaller than the number of sensors. The cost is not affected by the total size of the whole network, and is easily manageable for small values of the number of sources $r, K,\left|\mathcal{N}_{j}\right|, C$ and $T$.

\section{Some Remarks}

The two tracking algorithms proposed earlier have complimentary nature. Specifically, the one proposed in Sec. (3) is developed for a FC-based setting in which a central computing center receives and processes the data acquired at the sensors. A power constraint was introduced to ensure that the power of the signals to be transmitted does not exceed the total power budget imposed across all sensors. A different setting in which no FC is present, while only communication between neighboring sensors is allowed was considered and led to a totally distributed algorithm. The proposed joint source tracking and association scheme allows sensors at a close distance (and thus efficient to communicate with) to collaborate and exchange information to identify and track the sources. The FC-based setting allows faster processing, though it does not scale well with the size of the network and is prone to FC failures. Such issues are resolved by the distributed setting, though at the expense of slower processing due to the nested ADMM iterations during each coordinate cycle. 


\section{Simulations}

Next, we test the tracking and association performance of the two proposed schemes and compare it with alternative approaches. In the numerical tests it is assumed that among the summands in (2) only one has strong amplitude whereas the rest are negligible. This pertains to a setting where only one source, say the $\rho$ th, is close to sensor ${ }_{430} S_{j}$ whereas the rest are sufficiently far, thus their contribution in $x_{j}(t)$ is very small. Given the exponential attenuation that vibration sources experience in (3), it suffices for the sources to be separated by a distance of the order of $20-30$ meters. Consider the $2 \mathrm{D}$ field $[0,60] \times[0,60] \mathrm{m}^{2}$ in which $p=35$ sensors are deployed randomly. In the numerical tests, $r=2$ scalar sources are present. The transition matrix in 11 is set 435 equal to $\mathbf{F}=\operatorname{diag}(0.95,0.95)$.

The $(j, \rho)$ entry of matrix $\mathbf{H}_{t}$ is created according to a Gaussian random variable with expectation $e^{-\alpha\left\|\mathbf{p}_{j}-\mathbf{l}_{\rho}(t)\right\|_{2}}$ and variance $10^{-4}$, i.e., $\mathbf{H}_{t}(j, \rho) \sim \mathcal{N}\left(e^{-\alpha\left\|\mathbf{p}_{j}-\mathbf{l}_{\rho}(t)\right\|_{2}}\right.$, $10^{-4}$ ), where $\mathbf{p}_{j}$ corresponds to the location of sensor $j$, while $\mathbf{l}_{\rho}(t)$ the position of the $\rho$ th source at time instant $t$ and $\alpha=0.15$. The sources are located such that sensors 16,27 observe source $s_{1}(t)$ and sensors 18 and 23 observe source $s_{2}(t)$. This test holds for a total of 120 seconds. The sources move roughly for $0.13 \mathrm{~m}$ per time instant $t$ on a straight line which results the attenuation coefficients corresponding to sensors from which the sources move away to decrease to zero at a rate of approximately 0.98 , while the coefficients corresponding to sensors to which the sources approach closer, increase at a rate of $0.98^{-1}=1.02$. For instance, if source $\rho$ is moving away from sensor $j$ then $\mathbf{H}_{t}(j, \rho)$ decreases by the ratio $\frac{\mathbf{H}_{t}(j, \rho)}{\mathbf{H}_{t-1}(j, \rho)}=\frac{e^{-\alpha \| \mathbf{p}_{j}-1} \mathbf{1}_{(t) \|_{2}}}{e^{-\alpha \| \mathbf{p}_{j}-1 \rho^{(t-1) \|_{2}}}}=0.98$ since the source moves $\left\|\mathbf{p}_{j}-\mathbf{l}_{\rho}(t)\right\|_{2}-\left\|\mathbf{p}_{j}-\mathbf{l}_{\rho}(t-1)\right\|_{2} \approx 0.13 m$ away from sensor $j$ per time instant $t$. Similarly a rate of increase of 1.02 can be obtained when a source is approaching a sensor. The sparsity-controlling coefficients $\lambda_{\rho}$ 's are set as $\lambda_{1}=0.005$,

${ }_{450} \lambda_{2}=0.005$. The state and measurement noise variances are both set to be $\sigma_{u}^{2}=1.25$ and $\sigma_{w}^{2}=1.25$.

The FC-based tracking scheme is tested first. The tracking root mean-square error(RMSE) versus time $t$ is depicted in Fig. 3. The smallest tracking RMSE is achieved from the $K$-lag Kalman Smoother (KS) (using all the data until time $t$ to provide an 

cases, the (true) $\mathbf{H}_{t}$ is available. These two schemes treating $\mathbf{H}_{t}$ as known will be used as benchmarks to compare with the novel methods proposed here. In detail, note that the FC-based scheme evaluates $K$ different state estimates for the sources' state vector $\left\{\mathbf{s}_{\tau}\right\}_{\tau=t-K}^{t}$ at time instant $t$, according to 9 . Among these $K$ estimates, a filtered estimate (no delay) $\check{\mathbf{s}}_{t, t}$ is also provided (depicted as the green dashed-dot curve in Fig. 3). The performance of these estimates is pretty close to the estimates provided from a standard KF using our estimated $\hat{\mathbf{H}}_{t}$ (red solid curve in Fig. 3). Though in the long run, among the three schemes that do no have available the true $\mathbf{H}_{t}$, our FC-based scheme using $\check{\mathbf{s}}_{t-K}$, (see blue curve with cross markers) achieves a better performance while the performance gap with the traditional KF (purple curve with ' $\mathrm{x}$ ' markers) is reduced with time.

Considering the same FC-based setting described earlier, here we consider a limited power budget. The same testing scenario with $p=35$ sensors monitored field is considered here. The average tracking RMSE for tracking two sources is depicted in Fig. 4 with state and observation noise variances both set equal to $\sigma_{u}^{2}=1.25$ and $\sigma_{w}^{2}=1.25$. The total power budget is set as $P_{t o t}=40$, resulting on average (50 Monte Carlo runs) of $86 \%$ of all the sensors being selected to transmit their data to the fusion center. Our approach is compared with a FC-based tracking scheme where $86 \%$ of the sensors is selected randomly (blue solid curve). Moreover, our approach is compared with a

$\mathrm{KF}$ scheme utilizing the true $\mathbf{H}_{t}$ and using all sensors' data which, not surprisingly, achieves the lowest tracking RMSE (black crossed curve). This will act as a benchmark. Note that our approach (red dashed curve in Fig. 4) is following relatively close the benchmark KF scheme using the true sensing matrix $\mathbf{H}$, while $86 \%$ of the sensors is utilized to respect the total power constraint. This implies that the sensor selection process proposed in Sec. 3.4 efficiently finds sensors that contain information about the underlying sources, while respecting the available power budget.

Fig. 5 shows the average steady-state RMSE versus the percentage of sensors being selected i) via the scheme in Sec. 3.4, and ii) randomly. Note that the steady-state RMSE achieved by our proposed sensor selection scheme outperforms the random selecting scheme different values of the portion of selected sensors. In fact the advantage 
of using the sensor selection scheme in Sec. 3.4 increases as fewer sensors are being selected, following closely the steady-state RMSE achieved using all sensors and having available the true $\mathbf{H}$. Thus, the proposed sensor selection scheme judiciously selects sensors to respect the total power budget without significantly compromising the steady-state tracking RMSE.

The tracking performance of the novel distributed tracking and association algorithm in Sec. 44 is tested next. Again $p=35$ sensors are considered, while there are $r=4$ sources. The state transition matrix is set as $\mathbf{F}=\operatorname{diag}(0.95,0.95,0.95,0.95)$. The observation matrix $\mathbf{H}_{t}$ is generated as described earlier. The sparsity-controlling coefficients $\lambda_{\rho}$ 's are set as $\lambda_{1}=0.008, \lambda_{2}=0.007, \lambda_{3}=0.006$ and $\lambda_{4}=0.005$, while the incremental step parameter $c$ in the ADMM scheme is set equal to 1.0. Fig. 6 depicts the average tracking root mean-square error of 150 Monte Carlo trials for different tracking schemes with $K=80, \sigma_{u}^{2}=3.25$ and $\sigma_{w}^{2}=4.25$. The following four tracking schemes are tested and compared: i) the distributed tracking scheme in Sec. 4 (blue with cross markers curve); ii) the FC-based approach described in Sec. 3 (black dashed curve); iii) the distributed tracking scheme in Sec. 4 where $\mathbf{H}$ is fixed to the true $\mathbf{H}_{0}$ and not recursively updated later on (red solid curve); and iv) the FC-based approach described in Sec. 3 where the true $\mathbf{H}_{t}$ is used when estimating the states (purple with ' $\mathrm{x}$ ' markers curve). The number of ADMM iterations in the i) and iii) schemes is set to $C=50$ per coordinate descent cycle. Clearly, the centralized FCbased methods (black dashed and purple with ' $\mathrm{x}$ ' markers curves) achieve better tracking performance than the distributed algorithm. Nevertheless, our distributed algorithm (blue with cross markers curve) will eventually stay fairly close to the FC-based tracking scheme when $\mathbf{H}_{t}$ is not known. This is to be expected since in the distributed setting it takes more time for the information to propagate through the network. When the distributed scheme in 4 loses its capability to estimate $\mathbf{H}_{t}$ (red solid curve) then performance deteriorates compared to the fully blown distributed version (blue with cross markers curve) which includes estimation of $\mathbf{H}$. The lowest tracking RMSE is achieved by the centralized tracking scheme while using the true sensing matrix $\mathbf{H}_{t}$ throughout the tracking period.

In the same network setting, it is examined next in Fig. 7 7 how the number of ADMM 
iterations affects the steady-state RMSE of the distributed tracking scheme in Sec. 4. Two different noise settings are considered: i) $\sigma_{u}^{2}=1.25, \sigma_{w}^{2}=1.25$ (low-noise setting); and ii) $\sigma_{u}^{2}=3.25, \sigma_{w}^{2}=4.25$ (high-noise setting). The more ADMM iterations are applied, the closer to the centralized performance the distributed scheme is getting at the expense of a higher computational and communication complexity. The steadystate RMSE versus the window length $K$ is depicted in Fig. 8 for the two different noise settings described earlier. It can be seen that the tracking error decreases with an increasing window length $K$. The performance gap between the distributed and centralized schemes is smaller in the low-noise case compared to the high-noise setting.

The probability of successfully recovering the support of $\mathbf{H}$ at $t=120 \mathrm{~s}$ versus the window size $K$ is plotted in Fig. 9 Note that if the window length is $K=50$ or less, the sensing matrix $\mathbf{H}$ is not recovered well. As the window length becomes larger, the support of the unknown sensing matrix $\mathbf{H}$ becomes more likely to be recovered. The proposed distributed tracking scheme in Sec. 4 keeps close to the FC-based centralized scheme especially when a relatively large window length (say, $K=80$ ) is selected, for a number of $C=50 \mathrm{ADMM}$ iterations. The probability of recovering the support of $\mathbf{H}$ at $t=120 \mathrm{~s}$ versus the number of ADMM iterations $C$ is also shown in Fig. 10 . The larger the number of ADMM iterations, the more likely to recover correctly the support of $\mathbf{H}$.

\section{Conclusions}

The paper considers the problem of jointly associating sources with sensors and subsequently tracking them in i) a fusion center based sensor network; and ii) an ad hoc network architecture. The algorithm proposed for the FC-based is exploiting sparsity in the sensing matrix along with a power constraint, to jointly estimate the support of the sensing matrix along with the source states. Coordinate descent techniques along with solving a linear program are the building stones of the proposed algorithm. In the ad hoc setting the alternating direction method of multipliers is utilized to fully distribute the joint tasks of association and source tracking across the sensors. Numerical tests demonstrate the potential of the proposed schemes. 
Appendix A: Starting from the cost equation in (8), and using a common $\mathbf{H}$ for all $\mathbf{H}_{\tau}$ as justified earlier the cost can be rewritten as follows:

$$
\begin{aligned}
& \sum_{\tau=t-K+1}^{t}\left(\mathbf{x}_{\tau}-\mathbf{H} \mathbf{s}_{\tau}\right)^{T} \mathbf{\Sigma}_{w}^{-1}\left(\mathbf{x}_{\tau}-\mathbf{H} \mathbf{s}_{\tau}\right)+\sum_{\tau=t-K+1}^{t}\left(\mathbf{s}_{\tau}-\mathbf{F} \mathbf{s}_{\tau-1}\right)^{T} \mathbf{Q}^{-1}\left(\mathbf{s}_{\tau}-\mathbf{F} \mathbf{s}_{\tau-1}\right) \\
&+\left(\mathbf{s}_{t-K}-\hat{\mathbf{s}}_{t-K}\right)^{T} \mathbf{M}_{t-K \mid t-K}^{-1}\left(\mathbf{s}_{t-K}-\hat{\mathbf{s}}_{t-K}\right)+\sum_{\rho=1}^{r} \lambda_{\rho}\left\|\mathbf{h}_{\rho}\right\|_{1} \\
&=\left(\mathbf{s}_{t-K}-\hat{\mathbf{s}}_{t-K}\right)^{T} \mathbf{M}_{t-K \mid t-K}^{-1}\left(\mathbf{s}_{t-K}-\hat{\mathbf{s}}_{t-K}\right)+\sum_{\rho=1}^{r} \lambda_{\rho}\left\|\mathbf{h}_{\rho}\right\|_{1} \\
&+\left(\mathbf{F} \mathbf{s}_{t-K}-\mathbf{s}_{t-K+1}\right)^{T} \mathbf{Q}^{-1}\left(\mathbf{F} \mathbf{s}_{t-K}-\mathbf{s}_{t-K+1}\right)+\ldots+\left(\mathbf{F} \mathbf{s}_{t-1}-\mathbf{s}_{t}\right)^{T} \mathbf{Q}^{-1}\left(\mathbf{F} \mathbf{s}_{t-1}-\mathbf{s}_{t}\right) \\
&+\left(\mathbf{x}_{t-K+1}-\mathbf{H} \mathbf{s}_{t-K+1}\right)^{T} \mathbf{\Sigma}_{w}^{-1}\left(\mathbf{x}_{t-K+1}-\mathbf{H} \mathbf{s}_{t-K+1}\right)+\ldots+\left(\mathbf{x}_{t}-\mathbf{H} \mathbf{s}_{t}\right)^{T} \mathbf{\Sigma}_{w}^{-1}\left(\mathbf{x}_{t}-\mathbf{H} \mathbf{s}_{t}\right) \\
&=\left(\mathbf{x}_{\alpha}-\mathbf{F}_{\alpha} \mathbf{s}_{\alpha}\right)^{T} \mathbf{Q}_{\alpha}^{-1}\left(\mathbf{x}_{\alpha}-\mathbf{F}_{\alpha} \mathbf{s}_{\alpha}\right)+\sum_{\rho=1}^{r} \lambda_{\rho}\left\|\mathbf{h}_{\rho}\right\|_{1}
\end{aligned}
$$

where $\mathbf{s}_{\alpha}, \mathbf{x}_{\alpha}, \mathbf{Q}_{\alpha}$, and $\mathbf{F}_{\alpha}$ are given in equations (9), (10), and (12), respectively. The gradient of (37) w.r.t $\mathbf{s}_{\alpha}$ is $\nabla_{\mathbf{s}_{\alpha}} J_{t, K}\left(\left\{\mathbf{s}_{\tau}\right\}_{\tau=t-K}^{t}, \mathbf{H}\right)=-2 \mathbf{F}_{\alpha}^{T} \mathbf{Q}_{\alpha}^{-1}\left(\mathbf{x}_{\alpha}-\mathbf{F}_{\alpha} \mathbf{s}_{\alpha}\right)$. By setting the derivative to zero, the result in (9) follows.

Appendix B: When it comes to update matrix $\mathbf{H}_{t}$, while fixing the state vectors to their most up-to-date values only the first and last term in (7) are relevant. The matrix $\mathbf{H}_{t}$ will be updated by minimizing (7) on an entry-wise manner. To this end, the vector $\mathbf{y}_{i, t}^{\varkappa-1}$ in (14) is formed to help isolate the optimization variable $h \equiv \hat{\mathbf{H}}(i, \rho)$ from same-row entries which are fixed to their most up-to-date values $\hat{\mathbf{H}}_{t}^{\varkappa-1}(i, \ell), \ell \neq \rho, \ell=$ $1, \ldots, r$. Thus, (13) is minimized on an entry-by-entry coordinate fashion.

The minimization problem in (13) is equal to

$$
\left.\hat{\mathbf{H}}_{t}^{\varkappa}(i, \rho):=\arg \min _{h} \sigma_{w}^{-2} \| \mathbf{y}_{i, t}^{\varkappa-1}-\check{\mathbf{s}}_{\rho, t} \cdot h\right) \|_{2}^{2}+\lambda_{\rho} t, \text { s. to }-t \leq h \leq t
$$

Introducing the Lagrangian multipliers $\nu_{1}$ and $\nu_{2}$, the derivative of the Lagrangian function w.r.t. $h$ is given by

$$
\nabla_{h} \mathcal{L}\left(h, \nu_{1}, \nu_{2}\right)=2 \sigma_{w}^{-2} h \check{\mathbf{s}}_{\rho, t} \cdot \check{\mathbf{s}}_{\rho, t}^{T}-2 \sigma_{w}^{-2} \mathbf{y}_{i, t}^{\varkappa-1} \cdot \check{\mathbf{s}}_{\rho, t}^{T}+\nu_{1}-\nu_{2}
$$

After applying the KKT optimality conditions [4], it follows readily that the optimal solution of (38) is given by (15). 
Appendix C: The cost function in (23) can be rewritten as follows:

$$
\begin{aligned}
& \sum_{j=1}^{p} \sum_{\tau=t-K}^{t}\left(\xi_{1}(\tau) x_{j}(\tau)-\xi_{1}(\tau) \mathbf{H}_{j:} \mathbf{s}_{\tau, j}\right)^{T} \boldsymbol{\Sigma}_{w}^{-1}\left(\xi_{1}(\tau) \mathbf{x}_{j}(\tau)-\xi_{1}(\tau) \mathbf{H}_{j:} \mathbf{s}_{\tau, j}\right) \\
& +\frac{1}{p} \sum_{j=1}^{p} \sum_{\tau=t-K}^{t}\left(\xi_{1}(\tau) \mathbf{s}_{\tau, j}-\xi_{1}(\tau) \mathbf{F} \mathbf{s}_{\tau-1, j}\right)^{T} \mathbf{Q}^{-1}\left(\xi_{1}(\tau) \mathbf{s}_{\tau, j}-\xi_{1}(\tau) \mathbf{F} \mathbf{s}_{\tau-1, j}\right) \\
& +\frac{1}{p} \sum_{j=1}^{p} \sum_{\tau=t-K}^{t}\left(\xi_{2}(\tau) \mathbf{s}_{\tau, j}-\xi_{2}(\tau) \hat{\mathbf{s}}_{t-K, j}\right)^{T} \mathbf{M}_{t-K \mid t-K, j}^{-1}\left(\xi_{2}(\tau) \mathbf{s}_{\tau, j}-\xi_{2}(\tau) \hat{\mathbf{s}}_{t-K, j}\right) \\
& =\sum_{j=1}^{p} \sum_{\tau=t-K}^{t}\left(\xi_{1}(\tau) x_{j}(\tau)-\xi_{1}(\tau) \mathbf{H}_{j:} \mathbf{s}_{\tau, j}\right)^{2} \sigma_{w}^{-2} \\
& +\sum_{j=1}^{p} \sum_{\tau=t-K}^{t}\left\|p^{-1 / 2} \mathbf{Q}^{-1 / 2}\left(\xi_{1}(\tau) \mathbf{s}_{\tau, j}-\xi_{1}(\tau) \mathbf{F} \mathbf{s}_{\tau-1, j}\right)\right\|_{2}^{2} \\
& +\sum_{j=1}^{p} \sum_{\tau=t-K}^{t}\left\|p^{-1 / 2} \mathbf{M}_{t-K \mid t-K, j}^{-1 / 2}\left(\xi_{2}(\tau) \mathbf{s}_{\tau, j}-\xi_{2}(\tau) \hat{\mathbf{s}}_{t-K, j}\right)\right\|_{2}^{2} \\
& =\sum_{j=1}^{p} \sum_{\tau=t-K}^{t}\left\|\left[\begin{array}{c}
\xi_{1}(\tau) \sigma_{w}^{-1} x_{j}(\tau) \\
\xi_{1}(\tau)(p \mathbf{Q})^{-\frac{1}{2}} \mathbf{F} \mathbf{s}_{\tau-1, j} \\
\xi_{2}(\tau) p^{-1 / 2} \mathbf{M}_{t-K \mid t-K, j}^{-\frac{1}{2}} \hat{\mathbf{s}}_{t-K, j}
\end{array}\right]-\left[\begin{array}{c}
\xi_{1}(\tau) \sigma_{w}^{-1} \mathbf{H}_{j:} \\
\xi_{1}(\tau)(p \mathbf{Q})^{-\frac{1}{2}} \\
\xi_{2}(\tau) p^{-1 / 2} \mathbf{M}_{t-K \mid t-K, j}^{-\frac{1}{2}}
\end{array}\right] \cdot \mathbf{s}_{\tau, j}\right\|_{2}^{2}
\end{aligned}
$$

Appendix D: Differentiating the augmented Lagrangian in 27, w.r.t $\mathbf{s}_{\tau, j}$ gives

$$
\begin{aligned}
& \nabla_{\mathbf{s}_{\tau, j}} \mathcal{L}_{a}=-2 \mathbf{G}_{\tau, j}^{T}\left(\boldsymbol{\xi}_{\tau, j}-\mathbf{G}_{\tau, j} \mathbf{s}_{\tau, j}\right) \\
& \quad+\sum_{j^{\prime} \in \mathcal{N}_{j}}\left(\mathbf{v}_{\tau, j}^{j^{\prime}, k+1}(\kappa)+\omega_{\tau, j}^{j^{\prime}, k+1}(\kappa)\right)+c \sum_{j^{\prime} \in \mathcal{N}_{j}}\left(\mathbf{s}_{\tau, j}-\mathbf{z}_{\tau, j}^{j^{\prime}, k+1}(\kappa)\right)+c \sum_{j^{\prime} \in \mathcal{N}_{j}}\left(\mathbf{s}_{\tau, j}-\mathbf{z}_{\tau, j^{\prime}}^{j, k+1}(\kappa)\right)
\end{aligned}
$$

Setting the latter gradient equal to $\mathbf{0}$ (first-order optimality conditions), it follows for $j=1, \ldots, p$ and $\tau=t-K, \ldots, t$ that

$$
\begin{aligned}
& \mathbf{s}_{\tau, j}^{k+1}(\kappa+1)=\left(2 \mathbf{G}_{\tau, j}^{T} \mathbf{G}_{\tau, j}+2 c\left|\mathcal{N}_{j}\right| \mathbf{I}_{r \times r}\right)^{-1} \\
& \quad \times\left[2 \mathbf{G}_{\tau, j}^{T} \boldsymbol{\xi}_{j}-\sum_{j^{\prime} \in \mathcal{N}_{j}}\left(\mathbf{v}_{\tau, j}^{j^{\prime}, k+1}(\kappa)+\omega_{\tau, j}^{j^{\prime}, k+1}(\kappa)\right)+c \sum_{j^{\prime} \in \mathcal{N}_{j}}\left(\mathbf{z}_{\tau, j}^{j^{\prime}, k+1}(\kappa)+\mathbf{z}_{\tau, j^{\prime}}^{j, k+1}(\kappa)\right)\right]
\end{aligned}
$$

The gradient of the augmented Lagrangian function in 27 w.r.t $\mathbf{z}_{\tau, j}^{j^{\prime}}$ is

$$
\nabla_{\mathbf{z}_{\tau, j}^{j^{\prime}}} \mathcal{L}_{a}=c\left[\mathbf{z}_{\tau, j}^{j^{\prime}}-\mathbf{s}_{\tau, j}^{k+1}(\kappa+1)\right]+c\left[\mathbf{z}_{\tau, j}^{j^{\prime}}-\mathbf{s}_{\tau, j^{\prime}}^{k+1}(\kappa+1)\right]-\left[\mathbf{v}_{\tau, j}^{j^{\prime}, k+1}(\kappa)+\boldsymbol{\omega}_{\tau, j^{\prime}}^{j, k+1}(\kappa)\right]
$$

Thus, by setting the latter gradient equal to zero we obtain the updating formula in (34) for $j=1, \ldots, p, j^{\prime} \in \mathcal{N}_{j}$ and $\tau=t-K, \ldots, t$. 


\section{References}

\section{References}

[1] N. Ahmed, M. Rutten, T. Bessell, S. S. Kanhere, N. Gordon, and S. Jha, "Detection and Tracking Using Particle-Filter-Based Wireless Sensor Networks," IEEE Trans. on Mobile Computing, vol. 9, no. 9, pp. 1332-1345, Sep. 2010.

[2] I. F. Akyildiz, W. Su, Y. Sankarasubramaniam, and E. Cayirci, "Wireless Sensor Networks: A Survey," Computer Networks, Elsevier, vol. 38, pp. 393-422, 2002.

[3] B. D. O. Anderson and J. B. Moore, Optimal Filtering, Prentice Hall, Englewood Cliffs, NJ; 1979.

[4] D. P. Bertsekas, Nonlinear Programming, Second Edition, Athena Scientific, 2003.

[5] D P. Bertsekas, and J N. Tsitsiklis. Parallel and Distributed Computation: Numerical Methods. vol. 23. Englewood Cliffs, NJ: Prentice hall, 1989.

[6] S. Boyd, and L. Vandenberghe. Convex Optimization. Cambridge University Press, 2009.

[7] S. Boyd, N. Parikh, E. Chu, B. Peleato, and J. Eckstein. "Distributed Optimization and Statistical Learning via the Alternating Direction Method of Multipliers." Foundations and Trends in Machine Learning 3, no. 1, pp. 1-122, 2011.

[8] A. S. Charles and C. J. Rozell, "Dynamic Filtering of Sparse Signals using Reweighted $\ell_{1}$," Proc. of the Intl. Confe. on Acoust., Speech and Sig. Proc., Vancouver, CAN, pp. 6451-6455, May 2013.

[9] S. Cui, J. J. Xiao, A. J. Goldsmith, Z. Q. Luo, and H. V. Poor, "Estimation Diversity and Energy Efficiency in Distributed Sensing," IEEE Transactions on Signal Processing, vol. 55, no. 9, pp. 4683-4695, 2007.

[10] V. P. Dayan and K. N. Vijeyakumar, "Target Tracking in Sensor Networks Using Energy Efficient Prediction Based Clustering Algorithm," Procedia Engineering, vol. 38, pp. 2070-2076, 2012.

[11] S. S. Dias and M. G. S. Bruno, "Cooperative Target Tracking using Decentralized Particle Filtering and RSS Sensors," IEEE Trans. on Sig. Proc., vol. 61, no. 14, pp. 3632-3646, July 2013.

[12] R. Deming, J. Schindler and L. Perlovsky, "Multi-Target/Multi-Sensor Tracking Using Only Range and Doppler Measurements," IEEE Tans. on Aerospace and Electronic Systems, vol. 45, no. 2, pp. 593-611, April 2009. 
[13] P. Djuric, J. H. Kotecha, J. Zhang, Y. Hang, T. Ghirmai, M. F. Bugallo, and J. Miguez, "Particle Filtering," IEEE Signal Processing Magazine, vol. 20, no. 5, pp. 19-38, Sep. 2003.

[14] A. Doucet, B.-N. Vo, C. Andrieu, and M. Davy, "Particle Filtering for MultiTarget Tracking and Sensor Management," Proc. of the Fifth Intl. Conf. on Information Fusion, Annapolis, Maryland, July 2002, pp. 474-481.

[15] G. H. Golub and C. F. V. Loan, Matrix Computations, 3rd ed. Baltimore, MD: The John Hopkins Univ. Press, 1996.

[16] A. Gorji and M. B. Menhaj, "Multiple Target Tracking for Mobile Robots Using the JPDAF Algorithm," Proc. of IEEE Intl. Conf. on Tools with Artificial Intelligence, Patras, Greece, Oct. 2007, pp. 137-145.

[17] T. G. Gutowski and C. L. Dym, "Propagation of Ground Vibrations: A Review," Journal of Sound and Vibration, vol. 49, no. 2, pp. 179-193, 1976.

[18] R. A. Horn and C. R. Johnson, Matrix Analysis. Cambridge, U.K.: Cambridge Univ. Press, 1985.

[19] F. Jiang, J. Chen, and A. L. Swindlehurst, "Optimal Power Allocation for Parameter Tracking in a Distributed Amplify-and-Forward Sensor Network," arXiv preprint arXiv: 1309.3591, 2013.

[20] E. Karseras, K. Leung and W. Dai, "Tracking Dynamic Sparse Signals using Hierarchical Bayesian Kalman Filters," Proc. of the IEEE Intl. Conf. on Acoustics, Speech and Sig. Proc. (ICASSP), Vancouver, CAN, pp. 6546-6550, May 2013.

[21] S. M. Kay, Fundamental of Statistical Signal Processing: Estimation Theory, Prentice Hall, 1993.

[22] D. Y. Kim and M. Jeon,"Robust Distributed Kalman Filter for Wireless Sensor Networks with Uncertain Communication Channels," Mathematical Problems in Engineering, vol. 2012, Article ID 238597.

[23] D. Li, K. D. Wong, Y. H. Hu and A. M. Sayeed, "Detection, Classification, and Tracking of Targets," IEEE Sig. Proc. Mag., vol. 19, no. 2, pp. 17-29, March 2002.

[24] X. Lihua, S. Yeng-Chai, and C. E. De Souza, "Robust Kalman Filtering for Uncertain Discrete-Time Systems," IEEE Trans. on Automatic Control, vol. 39, no. 6, pp. 1310-1314, Jun. 1994.

[25] J. Lin, W. Xiao, F. L. Lewis, and L. Xie, "Energy-Efficient Distributed Adaptive 
Multisensor Scheduling for Target Tracking in Wireless Sensor Networks," IEEE Tr. on Instr. and Meas., vol. 58, pp. 1886-1896, 2009.

625
[35]

26] W. Ng, J. Li, S. Godsill, and J. Vermaak, "A Hybrid Approach for Online Joint Detection and Tracking for Multiple Targets," Proc. of the IEEE Aerospace Conference, Big Sky, MT, March 2005, pp. 2126-2141.

[27] S. Oh, "A Scalable Multi-Target Tracking Algorithm for Wireless Sensor Networks," International Journal of Distributed Sensor Networks, 2012.

[28] R. Olfati-Saber,"Distributed Kalman Filter With Embedded Consensus Filters," Proc. 44th Conf. Dec., the Eur. Control Conf., Seville, Spain, Dec. 2005, pp. 81798184.

[29] O. Ozdemir, R. Niu, and P. K. Varshney, "Tracking in Wireless Sensor Networks Using Particle Filtering: Physical Layer Considerations," IEEE Trans. on Signal Processing, vol. 57, no. 5, pp. 1987-1999, May 2009.

[30] W. J. Qi, P. Zhang and Z. L. Deng,"Weighted Fusion Robust Steady-State Kalman Filters for Multisensor System with Uncertain Noise Variances," Journal of Applied Mathematics, vol. 2014, Article ID 369252.

[31] G. Ren and I. D. Schizas, "Joint Sensors-Sources Association and Tracking under Power Constraint,"Proc. of the IEEE Global Conf. on Signal and Information Processing, Atlanta, GA, pp. 754-758, Dec. 2014.

[32] I D. Schizas, A. Ribeiro, and G B. Giannakis. "Consensus in Ad Hoc WSNs with Noisy LinksPart I: Distributed Estimation of Deterministic Signals," IEEE Transactions on Signal Processing, vol. 56, no. 1, pp. 350-364, 2008.

[33] I. D. Schizas, "Distributed Informative-Sensor Identification via Sparsity-Aware Matrix Decomposition," IEEE Trans. on Sig. Proc., vol. 61, no. 18, pp. 4610-4624, Sep. 2013.

[34] R. F. Souto, J. Y. Ishihara, and G. Araujo Borges, "A Robust Extended Kalman Filter for Discrete-Time Systems with Uncertain Dynamics, Measurements and Correlated Noise," Proc. of the 2009 American Control Conference, St. Louis, MS, pp. 1888-1893, June 2009.

35] Y. Tian and H. Qi, "Target Detection and Classification Using Seismic Signal Processing in Unattended Ground Sensor Systems,"Proc. of the Intl. Conf. on Acoust., Speech and Sig. Proc., Orlando, FL, 2002. 
[36] R. Tibshirani, "Regression Shrinkage and Selection via the Lasso," Journal of the Royal Statistical Society, Series B, vol. 58, no. 1, pp. 267-288, 1996.

[37] R. Tharmarasa, T. Kirubarajan, A. Sinha, and T. Lang, "Decentralized Sensor Selection for Large-Scale Multisensor-Multitarget Tracking," IEEE Trans. on Aerospace and Electronic Systems, vol. 47, no. 2, pp. 1307-1324, April 2011.

38] N. Vaswani, "Kalman Filtered Compressed Sensing," Proc. of Intl. Conf. on Imag. Proc., San Diego, CA, pp. 893896, 2008.

[39] J. Vermaak, S. J. Godsill, and P. Perez, "Monte Carlo Filtering for Multi Target Tracking and Data Association," IEEE Transactions on Aerospace and Electronic Systems, vol. 41, no, 1, pp. 309-332, 2005.

[40] L. Wang, Y. Zhao, W. Wang, "Study on Theory of Multi-target Tracking and Data Association Algorithms in Phased Array Radar,"Proc. of 6th Intl. Conf. on ITS, Chengdu, CH, pp. 1232-1235, 2006.

[41] X. Wang, D. Musicki, R. Ellem, and F. Fletcher, "Efficient and Enhanced MultiTarget Tracking with Doppler Measurements," IEEE Trans. on Aerospace and Electronic Letters, vol. 45, no. 4, pp. 1400-1417, Oct. 2009.

[42] L. Xie, Y. C. Soh, "Robust Kalman Filtering for Uncertain Systems," Systems \& Control Letters, vol. 22, no. 2, pp. 123-129, Feb. 1994.

[43] X. Yuanqing and H. Jingqing, "Robust Kalman Filtering for Systems Under Norm Bounded Uncertainties in all System Matrices and Error Covariance Constraints," Journal of Systems Science and Complexity, vol. 18, no. 4, pp. 339-445, Oct. 2005.

[44] Y. K. Zhan and A. K. S. Jardine, "Adaptive Autoregressive Modeling of NonStationary Vibration Signals Under Distinct Gear States. Part I: Modeling,” Journal of Sound and Vibration, vol. 286, pp. 429-450, 2005.

[45] H. Zhu, I. D. Schizas, and G. B. Giannakis, "Power-Efficient Dimensionality Reduction for Distributed Channel-Aware Kalman Tracking Using Wireless Sensor Networks," IEEE Tran. on Sig. Proc., vol. 57, no. 8, pp. 3193-3207, 2009.

[46] H. Zou, "The Adaptive Lasso and its Oracle Properties," Journal of the American Statistical Association, vol. 101, no. 476, pp. 1418-1429, 2006. 


\section{Figures}

1
1
2
3
4
4
5
6
7
7
8
9
9
10
11
12
13
14
15
16
16
17
18
19
29
21
29
22
23
24
24
25
26
27
27
28
29
30
31
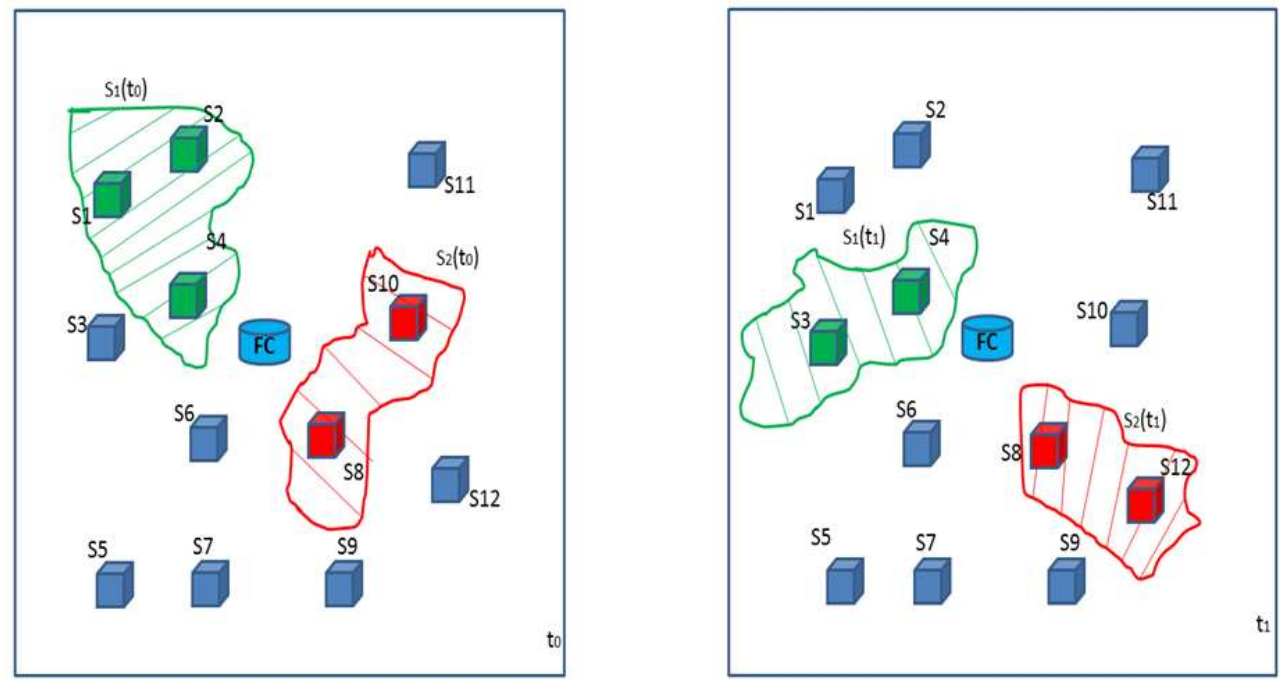

Figure 1: Time-varying fusion-center based multi-sensor multi-source configuration. 
50

51

52

53

54

55

56

57

58

59

60

61

62
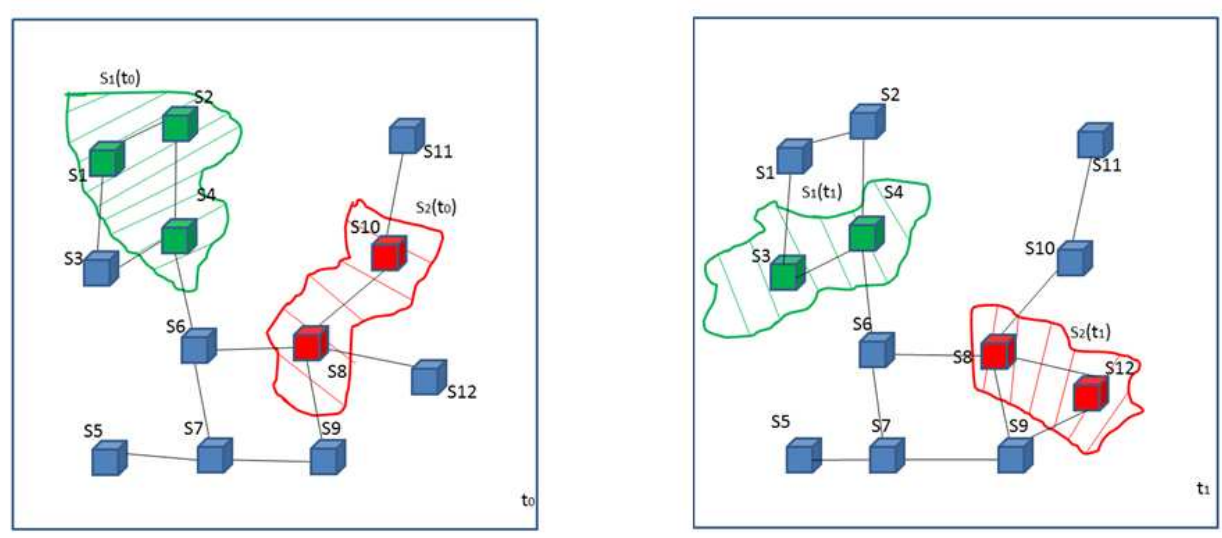

Figure 2: Ad hoc distributed time-varying multi-sensor multi-source configuration. 


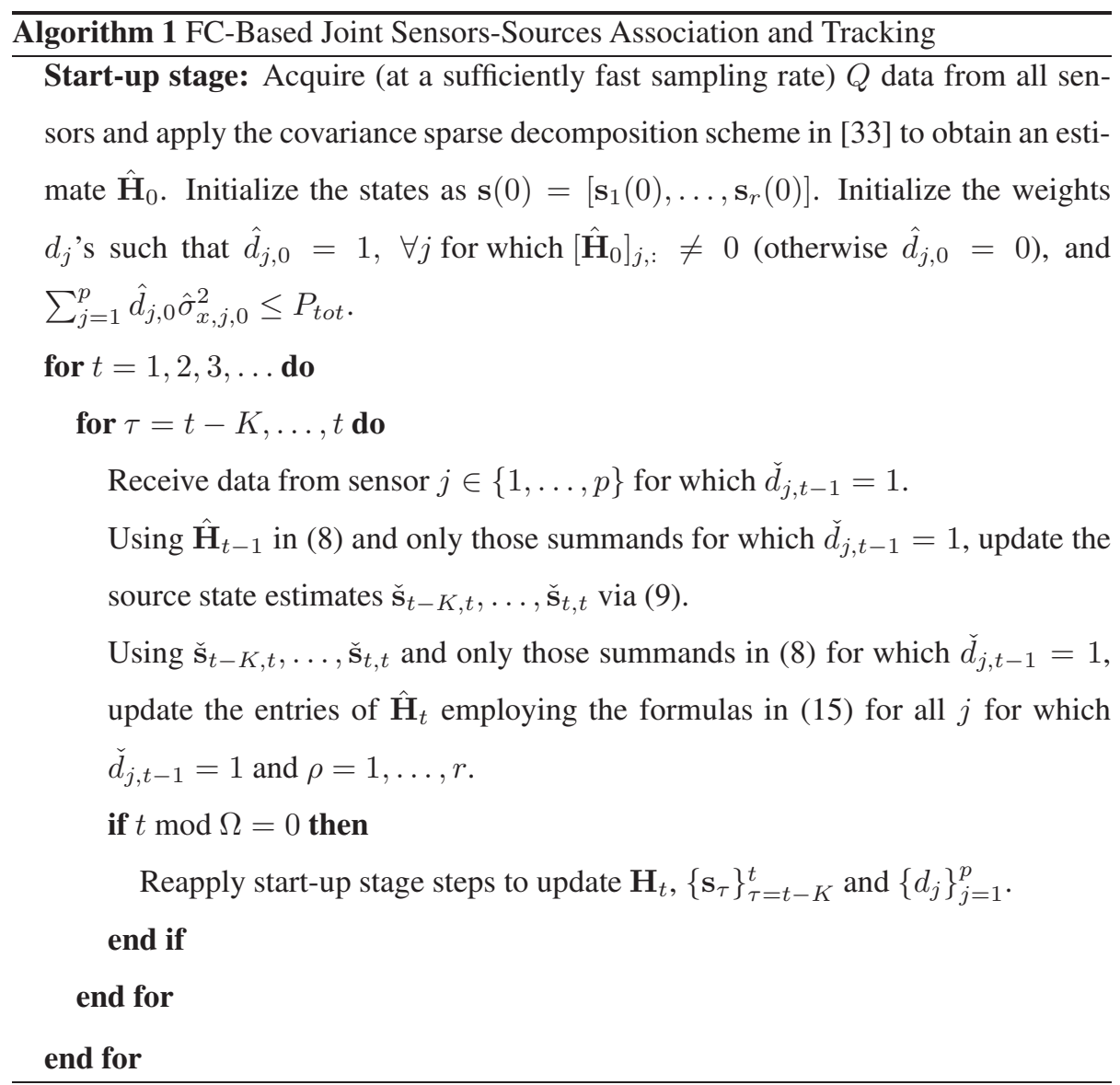




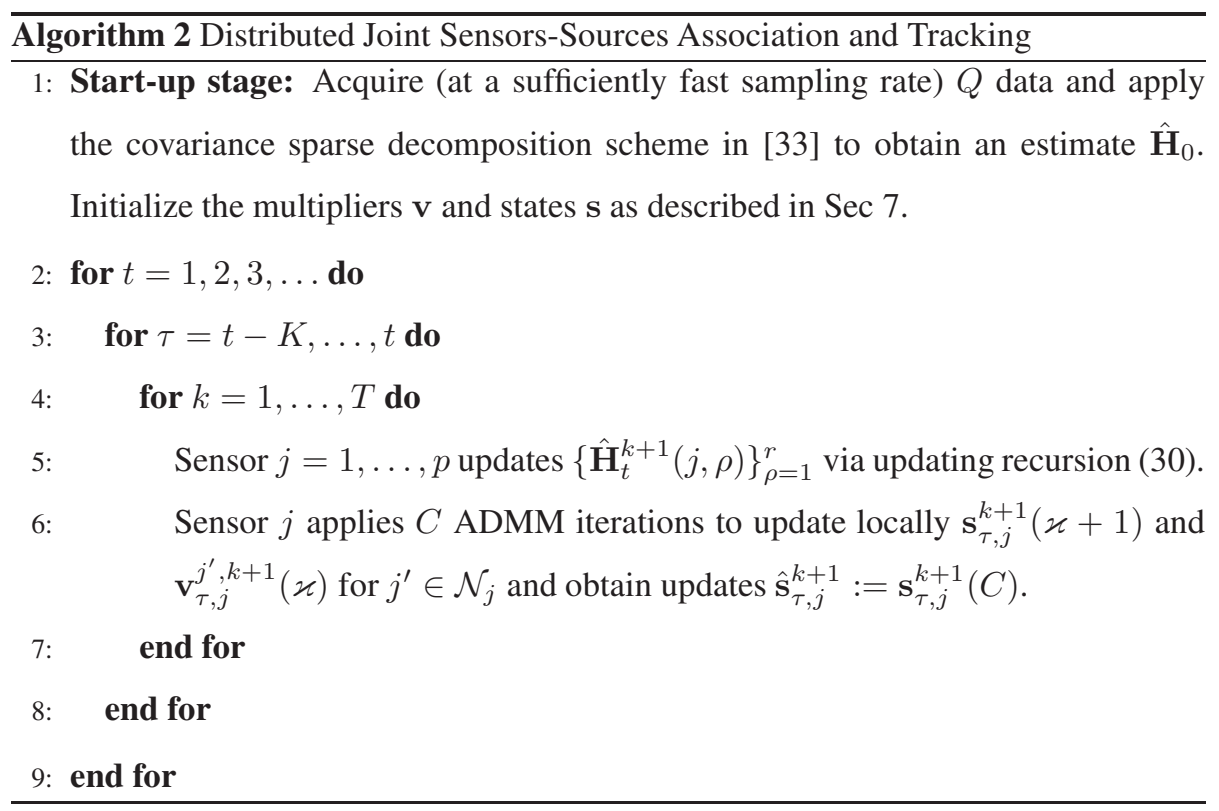




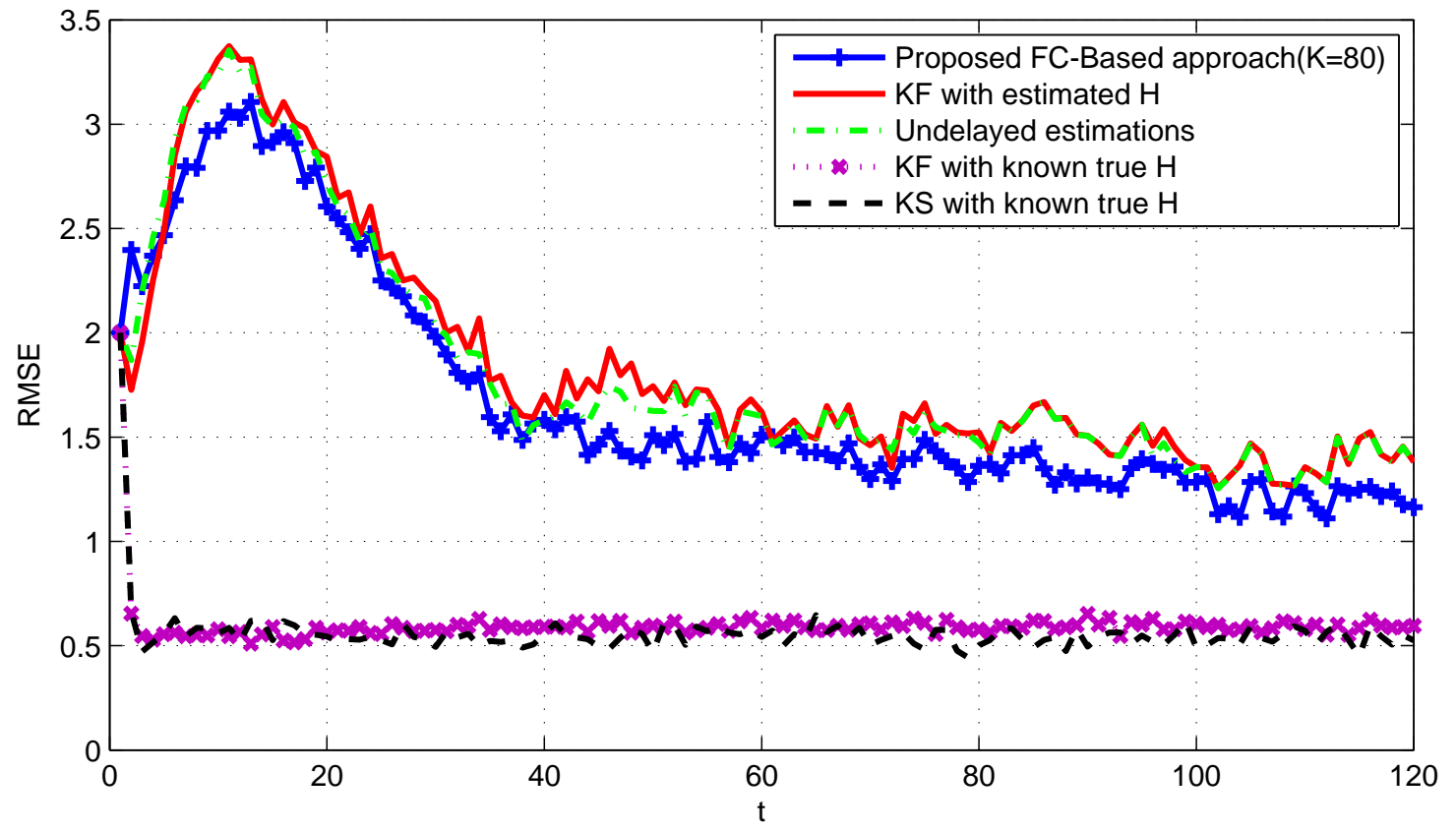

Figure 3: FC-based tracking root mean-square error (RMSE) vs. time $t$ 


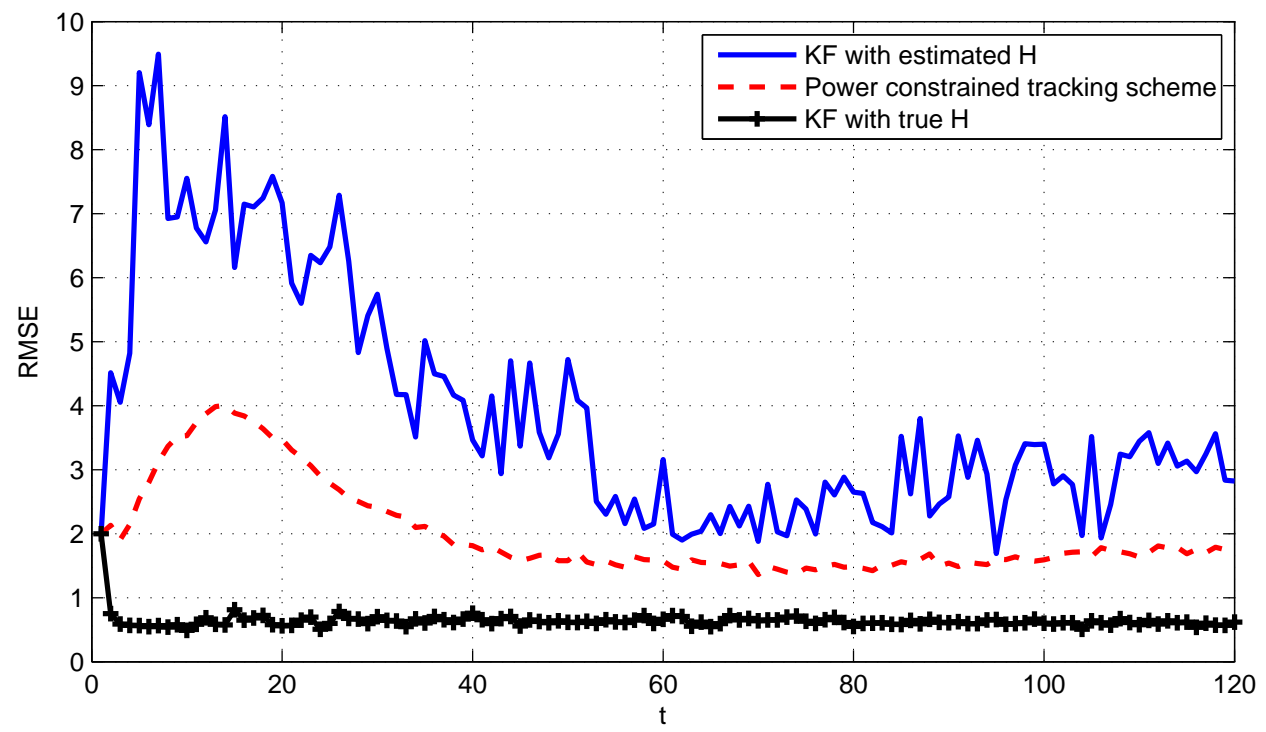

Figure 4: Tracking error vs. time $t$ under power constraints. 


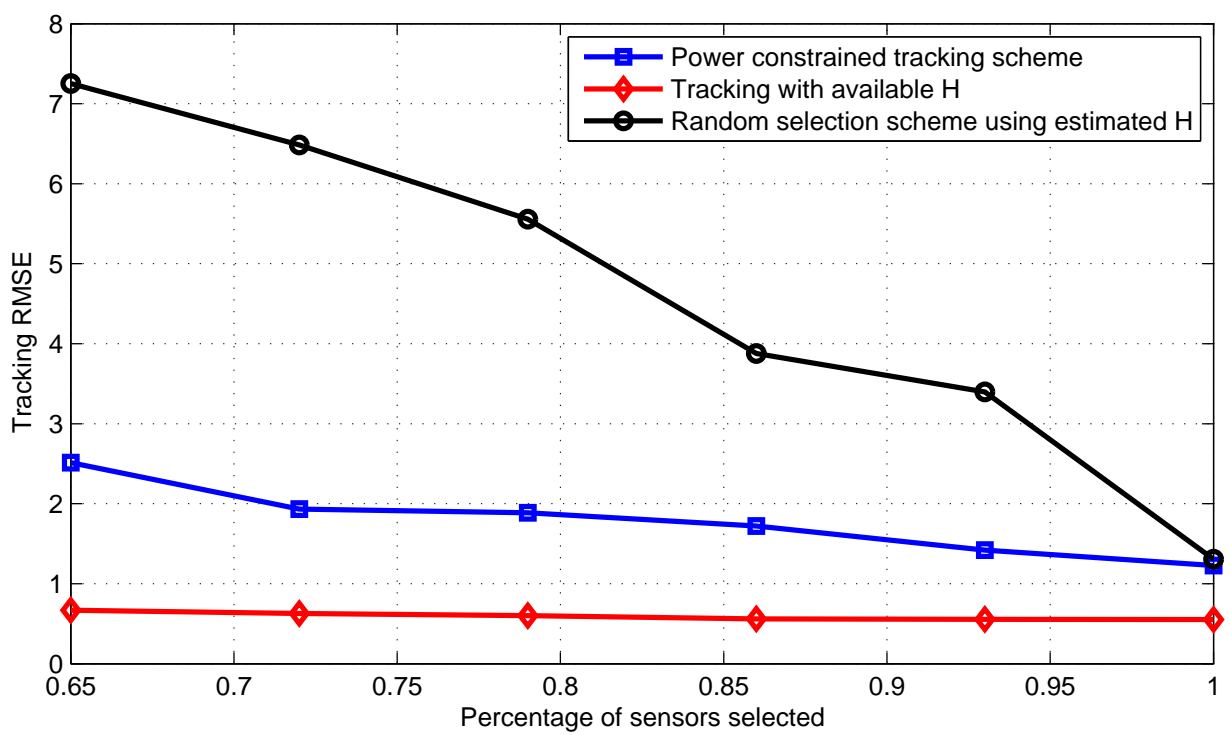

Figure 5: Steady-state root mean-square error (RMSE) vs. percentage of sensors selected 


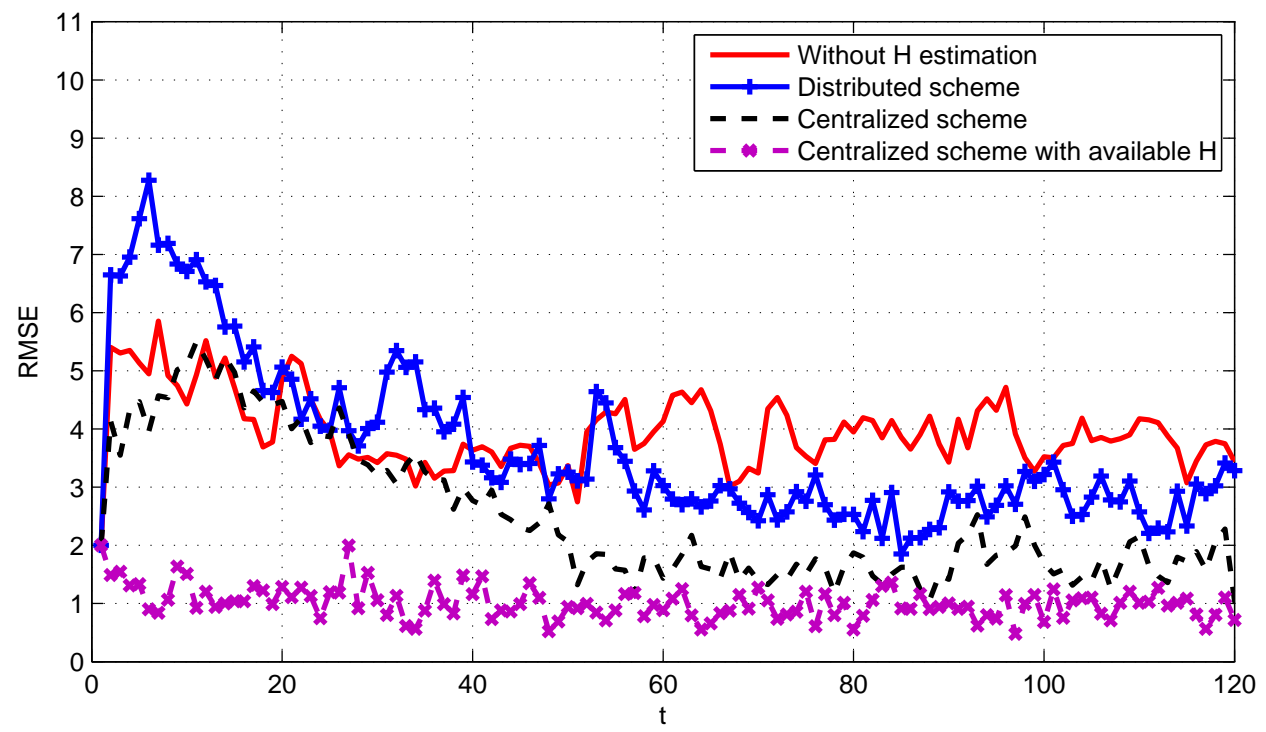

Figure 6: Tracking root mean-square error vs time $t$. 


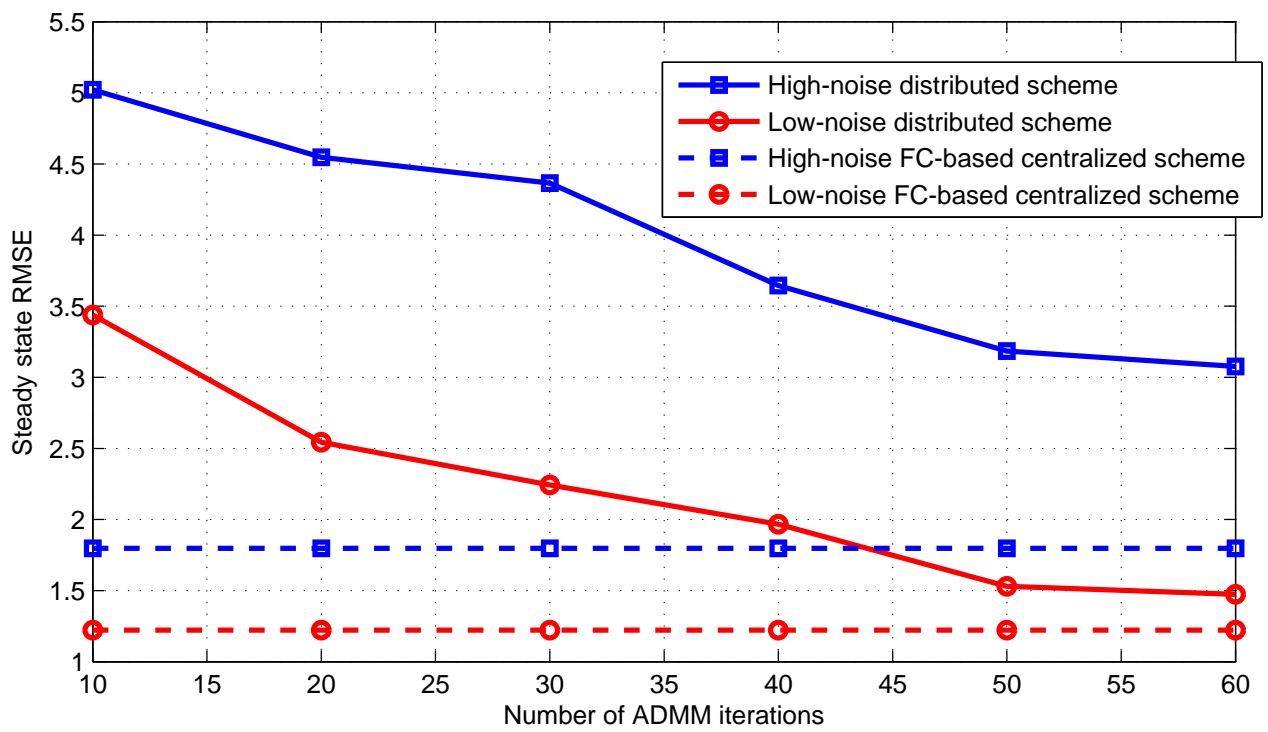

Figure 7: Steady-state root mean-square error (RMSE) vs. number of ADMM iterations. 


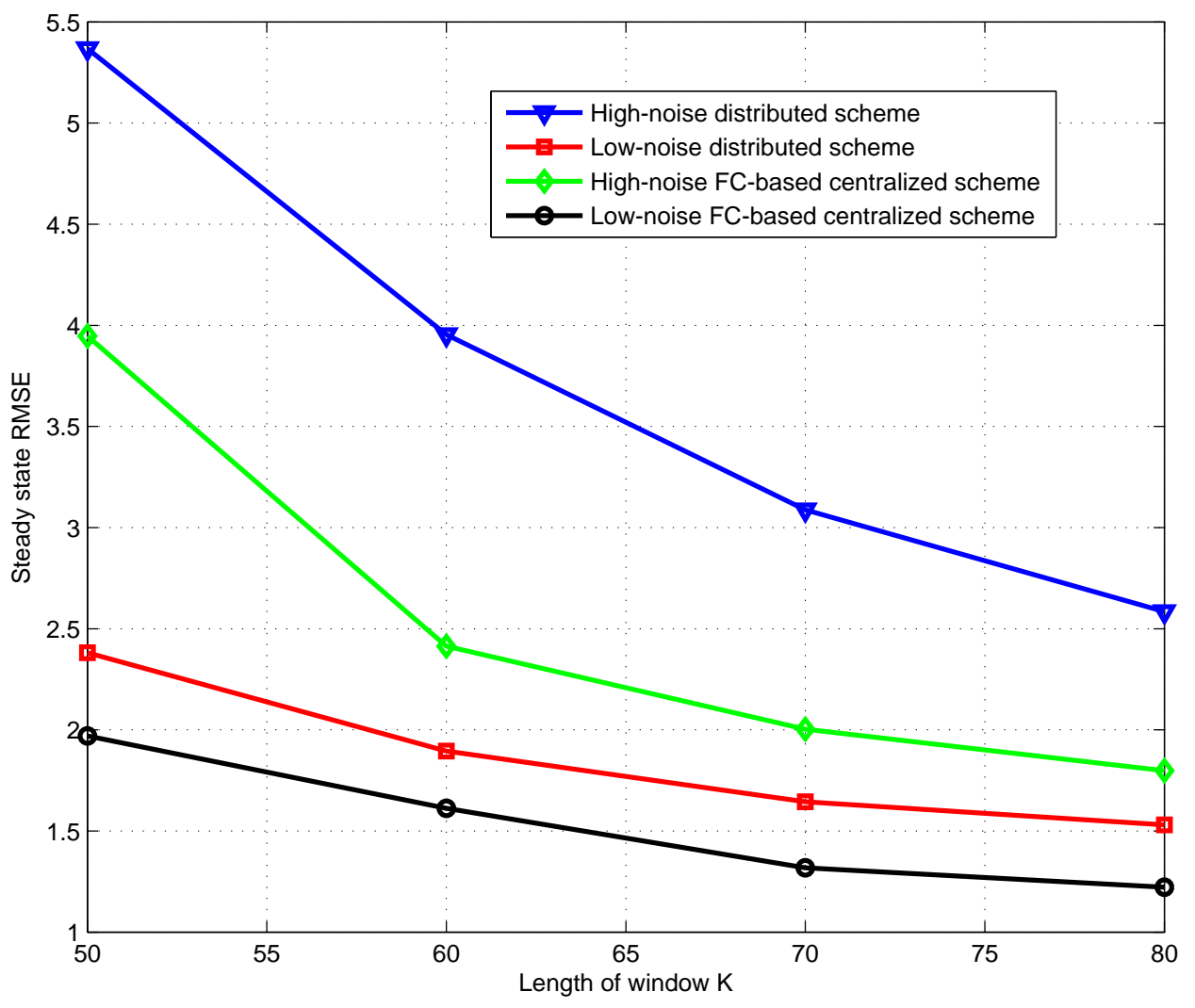

Figure 8: Steady-state root mean-square error (RMSE) vs. length of time window $K$. 


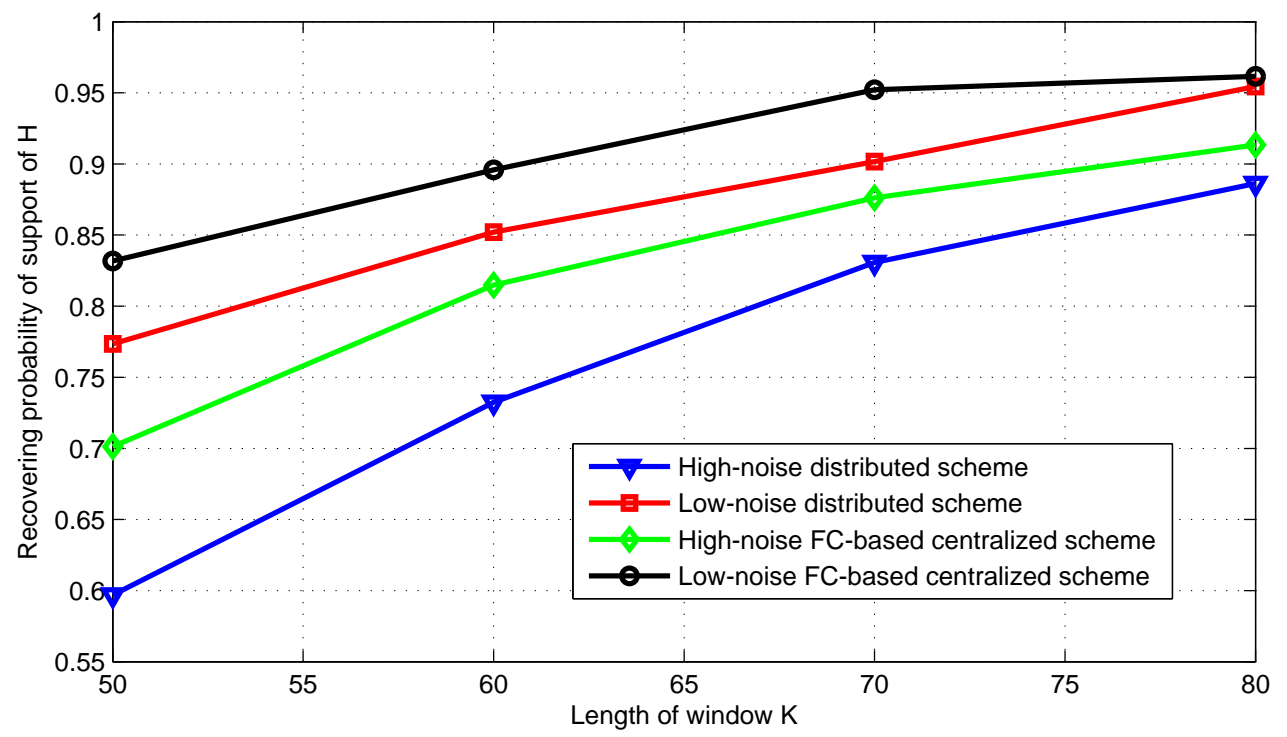

Figure 9: Probability of correctly recovering support of $\mathbf{H}$ vs. length of time window $K$. 


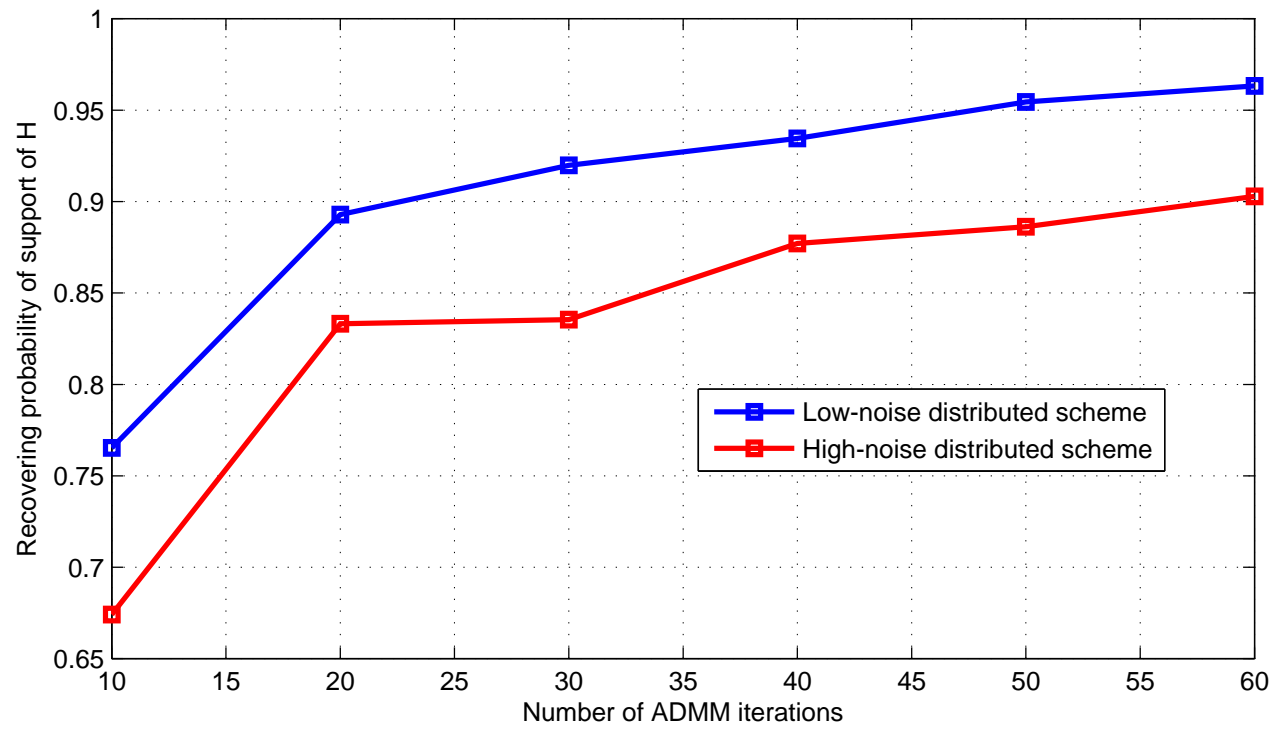

Figure 10: Probability of correctly recovering support of $\mathbf{H}$ vs. number of ADMM iterations. 